Chem. Ber. 119, 2025-2049 (1986)

\title{
Der Einfluß von Phenylgruppen auf die Homokonjugation im Bicyclo[3.2.1]octa-3,6-dien-2-yl-Anion. Eine ${ }^{13}$ C-NMR-Studie
}

\author{
Manfred Christl ${ }^{*}$ und Dieter Brückner ${ }^{1)}$ \\ Institut für Organische Chemie der Universität Würzburg, \\ Am Hubland, D-8700 Würzburg
}

Eingegangen am 10. Dezember 1985

Die Effekte von Deuteriumatomen in den Positionen 2 und 4 von exo-6-Brom- (11) und endo-6-Methoxytricyclo[3.2.1.0 2.7 $]$ oct-3-en (12) sowie exo-4-Methoxybicyclo[3.2.1]octa-2,6dien (13) auf deren ${ }^{13} \mathrm{C}$-chemische Verschiebungen stützen die frühere Interpretation der Effekte bei entsprechend deuterierten Bicyclo[3.2.1] octa-3,6-dien-2-yl-Anionen 1 und belegen so die homokonjugative Wechselwirkung in 1. - Ausgehend von Bicyclo[3.2.1] oct-6en-3-on (14) und Bicyclo[3.1.0]hex-2-en-endo-6-carbaldehyd (17) stellte man 3-Phenyl- (16) bzw. endo-4-Phenyl-(19a) und exo-4-Phenylbicyclo[3.2.1]octa-2,6-dien (19b) dar. 2,4-Diphenylbicyclo[3.2.1] octa-2,6-dien (6) und 19a, b wurden mit Methyllithium bzw. n-Butyllithium, Tricyclo[6.3.1.0 $\left.0^{2,7}\right]$ dodeca-2,4,6,9-tetraen (4) sowie 16 mit Kalium-tert-butoxid/n-Butyllithium deprotoniert, wobei die entsprechenden Bicyclo[3.2.1]octa-3,6-dien-2-yl-Anionderivate 7, $21 \mathrm{Li}, 5$ und 22 entstanden. Aus 19a,b wurde mit Kalium-tert-butoxid/nButyllithium auch $21 \mathrm{~K}$, d. h. das zugehörige Anion mit $\mathrm{K}^{+}$als Gegenion erhalten. Die NMR-Spektren von $21 \mathrm{Li}$ erwiesen sich im Gegensatz zu jenen von $21 \mathrm{~K}$ im Bereich von +45 bis $-30^{\circ} \mathrm{C}$ als temperaturabhängig. Die ${ }^{13} \mathrm{C}$-NMR-Spektren der Anionen und der zugehörigen Kohlenwasserstoffe werden im Vergleich mit den Spektren der unsubstituierten Verbindungen 1 und 2 ausführlich diskutiert. Insbesondere die starken Variationen der chemischen Verschiebungen von C-6,7 in Abhängigkeit von den Substituenten am Allylteil des Bicyclo[3.2.1] octa-3,6-dien-2-yl-Anionsystems bieten ein starkes Argument für die bishomoaromatische Natur dieser Anionen.

The Effect of Phenyl Groups on the Homoconjugation in the Bicyclo[3.2.1]octa-3,6-dien-2-yl Anion. A ${ }^{13}$ C NMR Study

The effects of deuterium atoms in the positions 2 and 4 of exo-6-bromo- (11) and endo-6methoxytricyclo[3.2.1.0.7. $]$ oct-3-ene (12) as well as exo-4-methoxybicyclo[3.2.1]octa-2,6diene (13) on their ${ }^{13} \mathrm{C}$ chemical shifts are in line with the previous interpretation of the effects in correspondingly deuterated bicyclo[3.2.1]octa-3,6-dien-2-yl anions 1, and thus provide evidence for the homoconjugative interaction in 1. 3-Phenylbicyclo[3.2.1] diene (16) and endo- (19a) as well as exo-4-phenylbicyclo[3.2.1]octa-2,6-diene (19b) have been prepared from bicyclo[3.2.1] oct-6-en-3-one (14) and bicyclo[3.1.0]hex-2-ene-endo-6carbaldehyde (17), respectively. 2,4-Diphenylbicyclo[3.2.1] octa-2,6-diene (6) and 19a, b were deprotonated by methyllithium and $n$-butyllithium, respectivley, whereas the proton abstraction from tricyclo[6.3.1.0 $0^{27}$ ]dodeca-2,4,6,9-triene (4) and from 16 required potassium tert-butoxide/ $n$-butyllithium. As products the corresponding bicyclo[3.2.1]octa-3,6-dien2 -yl anion derivatives have been identified. By means of potassium tert-butoxide/ $n$-butyl: lithium $19 \mathrm{a}, \mathrm{b}$ were converted into $21 \mathrm{~K}$, i.e. the anion with $\mathrm{K}^{+}$as counterion. The NMR

(C) VCH Verlagsgesellschaft mbH, D-6940 Weinheim, 1986

0009-2940/86/0606-2025\$ 02.50/0 
spectra of $21 \mathrm{Li}$ turned out to depend upon the temperature in the range between +45 and $-30^{\circ} \mathrm{C}$ in contrast to those of $21 \mathrm{~K}$. The ${ }^{13} \mathrm{C}$ NMR spectra of the anions and of the corresponding hydrocarbons are discussed in detail in relation to the spectra of the unsubstituted species 1 and 2. In particular, the wide variations of the chemical shifts of C-6,7 depending upon the substituents of the allylic moiety of the bicyclo[3.2.1]octa-3,6-dien2-yl anion system give strong support for the bishomoaromatic nature of these anions.

\section{A. Stand der Kenntnis}

Das von Winstein eingeführte Konzept der Homoaromatizität ${ }^{2)}$ wurde bei kationischen Systemen überzeugend experimentell belegt und für einige Kationen neuerdings auch durch hochentwickelte theoretische Rechnungen untermauert ${ }^{3 a, b)}$. Dagegen bestehen kaum Aussichten, homokonjugative Wechselwirkungen zwischen neutralen $\pi$-Systemen mit ausschlieBlich gepaarten Elektronen (closed-shell $\pi$ : systems) aufzufinden ${ }^{36}$. Bei geeignet substituierten Semibullvalenen und Barbaralanen trat kürzlich neben extrem kleinen Barrieren der degenerierten Cope-Umlagerung eine Temperaturabhängigkeit der UV-Spektren zutage $^{4}$, die große Hoffnungen bezüglich eines homoaromatischen Charakters weckt, der auf der Wechselwirkung zweier neutraler $\pi$-Systeme mit ungerader Elektronenzahl (open shell $\pi$ systems) beruht ${ }^{5)}$.

Als Prototyp anionischer Homoaromatizität galt seit den Arbeiten von Brown und Occolowit $z^{6)}$ sowie von Winstein et al. ${ }^{7}$ das Titelanion 1. Die Annahme der Delokalisierung der negativen Ladung über beide $\pi$-Systeme von 1 stützte sich auf den basenkatalysierten H/D-Austausch, dem die Allyl-H-Atome in Bicyclo[3.2.1]

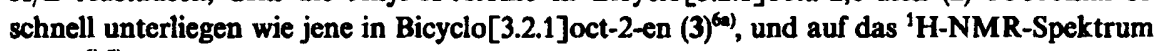
von $1^{6 b, 7)}$, in dem die Signale von 6,7-H und den beiden $8-\mathrm{H}$ im Vergleich zu den entsprechenden Signalen von 2 stark nach hohem Feld verschoben sind (vgl. Tab. 1 und 3). Die letzteren Effekte betrachtete man als Hinweise auf negative Partialladungen an C-6,7 bzw. auf einen aromatischen Ringstrom, der die Abschirmung der H-Atome an C-8 bewirken sollte.

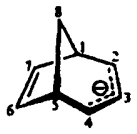

1<smiles>CCC1=CC2C=CC(C2)C1CC</smiles>

6

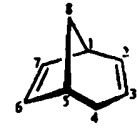

2<smiles>CCC1CC2C=CC1C2C</smiles>

7

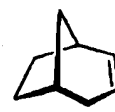

3

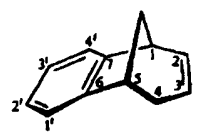

4<smiles>C1=CC2CCC(C1)C2</smiles>

9

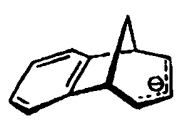

5

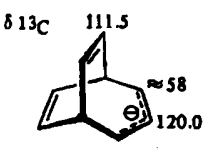

10

Der basenkatalysierte Austausch der Allyl-H-Atome der benzanellierten Verbindung 4 läuft nur mit ca. einem Neuntel der bei 2 ermittelten Geschwindigkeit, aber immer noch mehr als $3000 \mathrm{mal}$ so schnell ab wie in 3 . Daher wurde auf eine homokonjugative Stabilisierung auch im zugehörigen Anion 5 geschlossen ${ }^{8)}$. Andererseits fanden Trimitsis et al., daß kinetische $^{9 \mathrm{a})}$ und thermodynamische Acidität ${ }^{9 c)}$ von 4-H des Diphenyl-Derivats 6 von 2

Chem. Ber. 119 (1986) 
gegenüber 2,4-Diphenylbicyclo[3.2.1] oct-2-en kaum erböht sind, obwohl im ${ }^{1} \mathrm{H}$ - und ${ }^{13} \mathrm{C}$ NMR-Spektrum die Absorptionen der Atome in den Positionen 6, 7 und 8 des Anions 7 im Vergleich zu jenen von 6 beachtlich nach höheren Feld gewandert sind (vgl. Tab. 1 -4). Die Autoren verneinen daher die Frage nach der Homoaromatizität von 7.

Hohe kinetische Aciditäten, nur wenig geringer als die von 2, ermittelte man auch bei den Kohlenwasserstoffen $8^{10 a c)}$ und $9^{10 b, c)}$. Sie stehen im Einklang mit den $\mathrm{p} K_{\mathrm{a}}$-Werten der 4Positionen von 2, 8 und 9, die Washburn ${ }^{11)} \mathrm{zu} 31.4,34.0$ und 32.0 bestimmte. AufschluBreich ist der Vergleich dieser $\mathrm{p}_{\mathrm{z}}$-Werte mit denen der zugehörigen Kohlenwasserstoffe, die keine Doppelbindung in einer Zweikohlenstoffbrücke haben, womit die Stabilität der ungestörten Allyl-Anionen abgeschätzt werden kann. Der $\mathrm{p} K_{\mathrm{a}}$-Wert von 3 liegt aber mit $>40.5$ außerhalb des Meßbereichs der verwendeten Methode und dürfte in der Nähe der Werte von Cyclohexen und Cyclohepten $(44-46)^{12}$ angesiedelt sein. Die hohe Basizităt derartiger cyclischer Allyl-Anionen manifestiert sich auch darin, daß bisher die Messung von NMR-Spektren nicht gelang, weil die üblichen und aus Löslichkeitsgründen erforderlichen Solventien $\left[D_{8}\right]$ Tetrahydrofuran und $\left[D_{10}\right]-1,2$-Dimethoxyethan sehr rasch Deuteronen übertragen, was auch unseren Erfahrungen entspricht.

Semiempirische (MINDO/3, MNDO) und ab initio (STO-3G)-MO-Rechnungen ergaben nun überraschend keine Anhaltspunkte auf bishomoaromatische und bicycloaromatische Wechselwirkungen in den Anionen 1, 10 und dem Anion von 8, d. h. die Orbitale der beiden ungesättigten Strukturelemente, insbesondere das HOMO des Allylteils und das $\pi^{*}$-Orbital der Doppelbindung zwischen C-6 und C-7, würden nicht miteinander mischen ${ }^{13}$. Die hohe Acidität der Allylsysteme von 2 und 8 lastete man dem induktiven Effekt der C-6/C-7Doppelbindung, bei 9 den C-6/C-7- und C-8/C-9-Doppelbindungen an. Eine Erklärung der Hochfeldeffekte in den NMR-Spektren wurde nicht gegeben. Ab initio-(STO-3G)-MO-Rechnungen anderer Autoren ${ }^{14)}$ bejahten im Gegensatz zu obigen Arbeiten den homoaromatischen Charakter von 1 sowie einen Ladungstransfer von C-2,4 nach C-6,7. Angesichts der großen Fortschritte, die bei der Berechnung anionischer Systeme in den letzten Jahren erzielt worden sind ${ }^{15}$, darf man aber bezweifeln, ob die publizierten Rechnungen ${ }^{13,14}$ zuverlässige Ergebnisse liefern konnten.
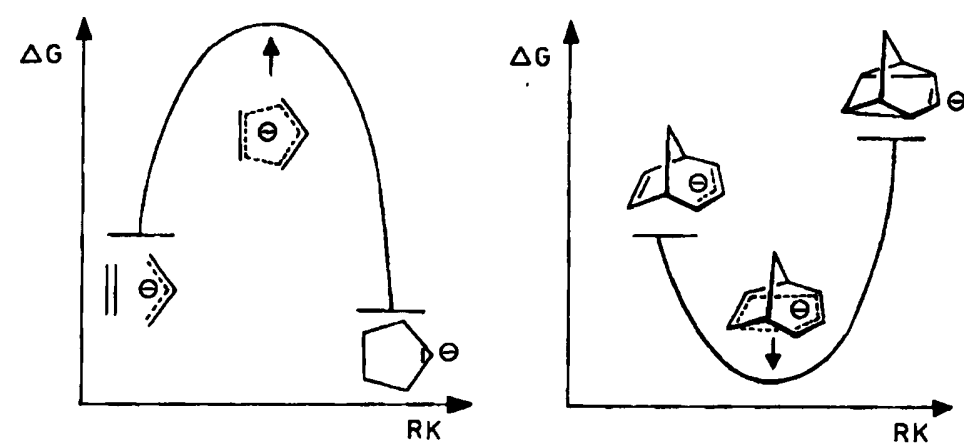

Abb. 1. Eniergiediagramme zur intermolekularen Wechselwirkung zwischen Allyl-Anion und Ethylen (links) und zur entsprechenden intramolekularen Wechselwirkung in 1 (rechts)

Schon die Woodward-Hoffmann-Regeln erlauben aufgrund der Orbitalsymmetrie eine Reaktion zwischen Allyl-Anion und Ethylen, wobei es sich um den elektronischen Prototyp der 1,3-dipolaren Cycloaddition ${ }^{16}$ handelt. Dem dabei durchlaufenen Ubergangszustand entspräche in 1 und seinen Analoga die bishomoaromatische Wechselwirkung. Dort wäre

Chem. Ber. 119 (1986) 
also der „Ubergangszustand" stabiler als „Ausgangszustand" und „Endprodukt" vom Cyclopentyl-Anion-Typ. Abb. 1 illustriert diese Fälle anhand von 1. Als Gründe für die nichtklassische Struktur von 1 ließen sich einerseits die Fixierung von Allyl-Anion- und EthylenEinheit in räumlicher, zur Wechselwirkung zwingender Nähe und andererseits die relativ hohe, durch die beiden Dreiringe bedingte Energie des „Produkts", des Tetracyclo[3.3.0.0 $\left.0^{28} \cdot 0^{4,6}\right]$ oct-3-yl-Anions, anführen.

Die ${ }^{13} \mathrm{C}-\mathrm{NMR}$-Spektroskopie ist zur Abschätzung von Ladungsverteilungen in Molekülen besonders gut geeignet ${ }^{17 a)}$. Goldstein et al. ${ }^{18)}$ publizierten die ${ }^{13} \mathrm{C}$-chemischen Verschiebungen des Anions 10. Zwar gleichen die Werte des Allylteils unter Berücksichtigung der Substitution jenen des unsubstituierten Allyl-Anions (23) ${ }^{19}$, aber das Signal von C-6,7,8,9 ist mit $\delta=111.5$ gegenüber den Werten, die man von C-6,7,8,9 im Kohlenwasserstoff 9 zu erwarten hat, um wenigstens $20 \mathrm{ppm}$ nach höherem Feld verschoben. Köhler und Hertkorn ${ }^{209}$ beschrieben das ${ }^{13} \mathrm{C}-\mathrm{NMR}$-Spektrum von 1 gleichzeitig mit uns ${ }^{21)}$ (Tab. 4). Die chemischen Verschiebungen des Allylteils stimmen fast überein mit jenen von 10, jedoch absorbieren $C$ 6,7 mit $\delta=91.02$ bei extrem hohem Feld. Im Vergleich zum Kohlenwasserstoff 2 (Tab. 2) betragen die Hochfeldeffekte 39.34 und $48.80 \mathrm{ppm}$. Sie wurden als Wirkung von zwei Faktoren interpretiert, erstens der Ladungsübertragung von C-2,4 nach C-6,7 und zweitens der Umhybridisierung von C-6,7 (und auch C-2,4) in Richtung $\mathrm{sp}^{3}$ infolge der homokonjugativen Wechselwirkung, die ja zur Erhōhung der Ligandenzahl von C-2,4,6,7 führen müßte ${ }^{21}$. Diese Erklärung würde den Unterschied der chemischen Verschiebungen von C-6,7 in 1 einerseits und $C-6,7,8,9$ in 10 andererseits verständlich machen, denn in 10 wäre die vom Allylteil ausgehende Wechselwirkung auf doppelt so viele C-Atome verteilt wie in 1. Tatsächlich ist die Summe der Hochfeldeffekte auf C-6,7 beim Ubergang $2 \rightarrow 1$ ähnlich wie jene auf C-6,7,8,9 beim Übergang $9 \rightarrow 10$. Tiefe Temperaturen und Lösungsmittelwechsel bei $1^{21)}$ und $10^{18)}$ sowie verschiedene Gegenionen $2010^{18)}$ änderten die chemischen Verschiebungen nur minimal.

Durch die ${ }^{13} \mathrm{C}-\mathrm{NMR}-$ Spektren von $[2-\mathrm{D}]-1$ und $\left[2,4-\mathrm{D}_{2}\right]-1$, die nur den sich streng additiv verhaltenden eigentlichen (intrinsic) Isotopeneffekt zeigen, wurde sichergestellt, daß 1 eine einheitliche Verbindung und nicht ein rasch äquilibrierendes Gemisch aus 1 ohne Wechselwirkung zwischen den $\pi$-Systemen und seinen tri- und tetracyclischen Isomeren ist. Weiterhin spricht die Größe des Deuteriumeffekts auf die chemische Verschiebung von C-6,7 für direkte Bindungsbeziehungen zwischen $\mathrm{C}-2$ und $\mathrm{C}-7$ sowie $\mathrm{C}-4$ und $\mathrm{C}-6^{21}$.

Wir stellen jetzt einige Modellverbindungen vor, deren Deuterium-Isotopeneffekte auf die ${ }^{13} \mathrm{C}$-chemischen Verschiebungen die Interpretation der Befunde bei $[2-\mathrm{D}]-1$ und $\left[2,4-\mathrm{D}_{2}\right]-$ ${ }^{21)}$ stützen und berichten über die Störung des Anions 1 durch Phenylsubstituenten in den Positionen 2, 3 und 4 und durch Benzoanellierung in den Positionen 6 und 7.

\section{B. Ergebnisse}

1. Deuterium-Isotopeneffekte auf die ${ }^{13} \mathrm{C}$-chemischen Verschiebungen von zwei Tricyclo[3.2.1.0 2,7] oct-3-enen $(11,12)$ und exa-4-Methoxybicyclo[3.2.1]octa-2,6-dien (13)

Die Verbindungen 11a-13a (Schema 1) waren als Vorstufen (11a, 13a) bzw. als Nebenprodukt (12a) bei der Synthese von $\left[2,4-D_{2}\right]-1$ angefallen ${ }^{21}$. Zur Darstellung gingen wir von $\left[2,4,4-D_{3}\right]-2^{6 a)}$ aus, das einen Anteil weniger deuterierten Materials enthielt, und kamen mit Hilfe von $N$-Bromsuccinimid in Tetrachlormethan nach der Vorschrift zur Umsetzung von nichtdeuteriertem $2^{22)}$ zu einem Gemisch der ungefähren Zusammensetzung 11a:11 b:11c $=8: 1: 1$. Die durch Silbernitrat katalysierte Methanolyse dieses Gemisches führte zu den drei bicycli-

Chem. Ber. 119 (1986) 
schen exo-Methylethern 13 (a:b:c $\approx 8: 1: 1$ ), den zugehörigen endo-Methylethern und den tricyclischen Methylethern 12 (a:b:c $\approx 8: 1: 1)$, die gaschromatographisch getrennt wurden. Zusammen mit der nichtdeuterierten Verbindung dienten 13a-c zur Bereitung einer Mischung aus 1, [2-D]-1 und $\left[2,4-D_{2}\right]-1^{21}$.

Aufgrund der Produktverteilung (vgl. exp. Teil) dürfte die Umwandlung von 11 nicht als $\mathrm{S}_{\mathrm{N}} 1-$ Reaktion ablaufen. Kirmse und $O$ olbricht ${ }^{23)}$ fanden nämlich, daß das von 11 abgeleitete Carbokation weit überwiegend den nicht umgelagerten exoMethylether liefert, den wir bei obigem Versuch nicht nachweisen konnten. So nehmen wir für die Bildung von 12 einen $\mathrm{Ag}^{+}$-unterstützten $\mathrm{S}_{\mathrm{N}}$ 2-Prozeß und für die von 13 und seinem Diastereomeren $\mathrm{Ag}^{+}$-unterstützte Homo-S $\mathrm{S}_{\mathrm{N}} 2^{\prime}$ - oder Homo$S_{\mathrm{N}} 2^{\prime \prime}$-Reaktionen an.

Schema $1 .{ }^{13} \mathrm{C}$-Chemische Verschiebungen $(\delta$-Werte) der deuterierten Verbindungen $11-13$ in $\mathrm{CDCl}_{3}$. Off-resonance-entkoppelte Spektren dienten als Zuordnungshilfe.

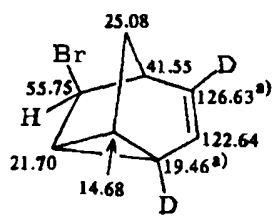

11 a

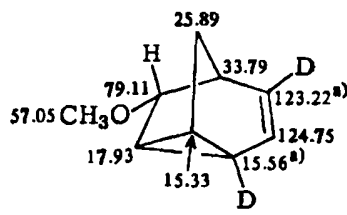

$12 \mathbf{a}$

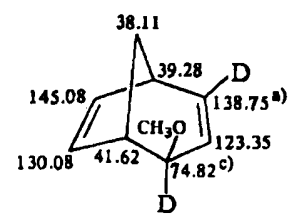

13

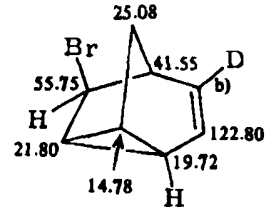

$11 \mathrm{~b}$

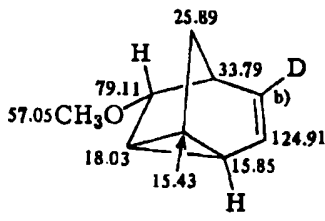

$12 \mathbf{b}$

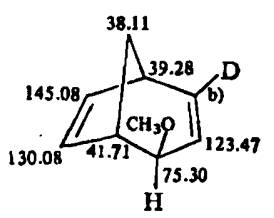

13b

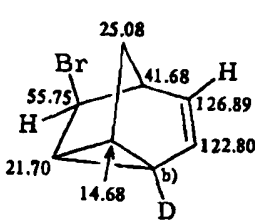

$11 \mathrm{c}$

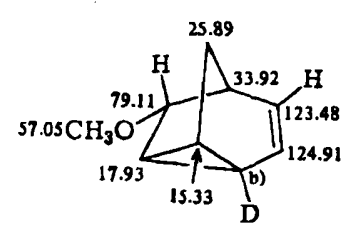

$12 \mathrm{c}$

$\mathrm{CH}_{3}$ jeweils 56.07

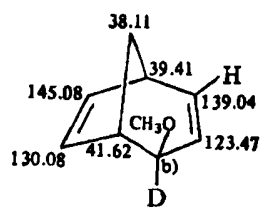

$13 \mathbf{c}$

a) $1: 1: 1-$ Triplett, $J_{C, D}=25-26 \mathrm{~Hz},-{ }^{b)}$ Signal wegen zu geringer

Intensität nicht beobachtet. ${ }^{c)} 1: 1: 1-$ Triplett, $J_{C, D}=23 \mathrm{~Hz}$.

Die ${ }^{13} \mathrm{C}$-NMR-Spektren von 11-13 wurden unter Routinebedingungen aufgenommen und sind daher nicht besser als bis 0.05 ppm aufgelöst. Die Banden der dideuterierten Spezies a tauchen ausnahmslos bei höchstem Feld auf, wenn sie sich lagemäßig von denen der monodeuterierten $b$ und $c$ unterscheiden. Da

Chem. Ber. 119 (1986) 
nur Isotopeneffekte über eine oder zwei Bindungen gefunden werden, ist die Zuordnung bei Berücksichtigung von Literaturdaten ${ }^{24}$ trivial. Besonders hervorgehoben sei der 0.10-ppm-Effekt der D-Atome der 2-Positionen in 11 a,c und 12a,c auf C-1 und C-7. Bei 1 betragen diese Größen 0.14 und $0.07 \mathrm{ppm}$ und deuten so die partiellen Bindungsbeziehungen C-2/C-7 und C-4/C-6 an. Dagegen wirken die D-Atome der 4-Positionen von $11 \mathrm{a}, \mathrm{b}$ und $12 \mathrm{a}, \mathrm{b}$ auf $\mathrm{C}-6 \mathrm{nicht}$, ebensowenig wie die D-Atome der 2- und 4-Positionen von 13 auf C-6 und C-7, was auf die fehlende direkte Bindungsbeziehung zwischen den betreffenden C-Atomen zurückgehen sollte. Somit bestätigen die Daten von 11 - 13 die Interpretation der Spektren von $[2-D]-1$ und $\left[2,4-D_{2}\right]-1^{21)}$.

\section{Darstellung von 3- (16) und 4-Phenylbicyclo[3.2.1]octa-2,6-dien (19)}

Die Ziel-Anionen 5, 7, 21 und 22 sollten durch Deprotonierung der zugehörigen Kohlenwasserstoffe 4, 6, 19 bzw. 16 mit Hilfe geeigneter Basen erzeugt werden. Trimitsis und Tuncay ${ }^{96, c)}$ hatten zur Umwandlung von 6 in $7 n$-Butyllithium benutzt. Bei den weniger aciden Verbindungen 4, 16 und 19 war die Anwendung von Kalium-tert-butoxid/ $n$-Butyllithium vorgesehen. Mit diesem Reagenz hatten Köhler und Hertkorn ${ }^{201} 1$ aus 2 erhalten und so die Bereitung von 1 erheblich vereinfacht, zu dem man früher ${ }^{60,7)}$ nur durch Spaltung reinen exo-4-Methoxybicyclo[3.2.1] octa-2,6-diens mit $\mathrm{Na} / \mathrm{K}$-Legierung gelangte.

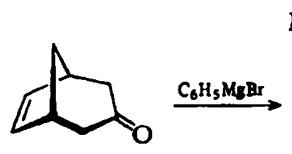

14<smiles>CCCCCCCCCCCCC1(O)CC2C=CC(C1)C2C</smiles>

15
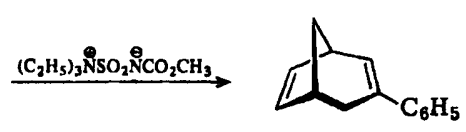

16

Die bislang unbekannten Kohlenwasserstoffe 3- (16) und 4-Phenylbicyclo[3.2.1]octa-2,6-dien (19) stellten wir aus dem Keton 14 bzw. dem Aldehyd 17 dar. Für 14, das auf verschiedenen Routen zugänglich ist, wählten wir den von LeBel und Liesemer ${ }^{25}$ ) eingeschlagenen Weg über 8-Bromtricyclo[3.2.1.0 ${ }^{27}$ ]octan-3-on, erhielten aber hauptsächlich den durch Überreduktion gebildeten, von 14 abgeleiteten Alkohol. Ein ähnliches Resultat erzielten Bos und Klumpp ${ }^{26}$ bei der Umwandlung von Tetracyclo[3.3.0.0.8. ${ }^{2,6}$ ] octan-3-on. Durch Oxidation des Reduktionsprodukts kamen wir dann zum gewünschten 14, das wir auch nach Klumpp et al. ${ }^{27}$ bereiteten. Phenylmagnesiumbromid überführte 14 in den Alkohol $15(82 \%$ Ausbeute), dessen Dehydratisierung zu 16 (42\% Ausbeute) mit dem BurgessReagenz ${ }^{28)}$ erreicht wurde. Die NMR-Spektren belegen die Strukturen von 15 und 16, jedoch ist die Konfiguration von 15 nicht bekannt.

Zur Synthese von 19 griffen wir auf die bewährte Strategie zurück, nach der aus Bicyclo[3.1.0]hex-2-en-endo-6-carbaldehyd (17) mit Hilfe einer Olefinierungsreaktion ein cis-Divinylcyclopropan-Derivat aufgebaut und dieses durch CopeUmlagerung in das gewünschte Bicyclo[3.2.1]octa-2,6-dien umgewandelt wird. Die Stammsubstanz ${ }^{299}$ sowie folgende Abkömmlinge gewann man auf diese Weise: 4,4-Dideuterio ${ }^{63)}$, 4-Methoxy ${ }^{6 b)}$, 4,4-Dimethyl ${ }^{30)}$, exo-4-Carbonsäure ${ }^{311}$. 


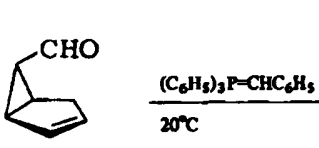

17

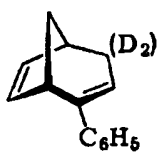

$20\left(\left[D_{2}\right]-20\right)$

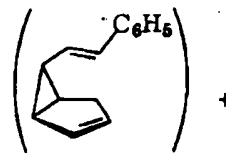

18 a<smiles>[13CH3]</smiles>

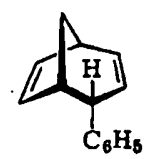

19a

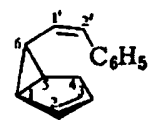

$18 b$<smiles>C1CCCC1</smiles><smiles>C1=CC2C=CC1C2</smiles>

$19 b$

Dementsprechend kamen wir ausgehend von 17 und Benzylidentriphenylphosphoran mit $28 \%$ Ausbeute $\mathrm{zu}$ einem 3:2-Gemisch von cis-Styrylbicyclo[3.1.0] hexen 18b und endo-4-Phenylbicyclo[3.2.1]octa-2,6-dien (19a) und nach Erhitzen auf $150^{\circ} \mathrm{C}$ zu einem 2:3-Gemisch aus endo- (19a) und exo-4-Phenylbicyclo[3.2.1] octa-2,6-dien (19b). Offenbar war aus der Wittig-Reaktion ein Gemisch der Bicyclen 18a,b mit $E$-bzw. Z-konfigurierter Seitenkette hervorgegangen und die $E$-Verbindung 18 a schon bei $20^{\circ} \mathrm{C}$ umgelagert. Dagegen blieb das $Z$-Isomere $18 \mathrm{~b}$ bei $20^{\circ} \mathrm{C}$ unverändert, wohl weil hier der sterische Anspruch der Phenylgruppe die Energie des Ubergangszustandes der Cope-Umlagerung anhebt. Einen derartigen Reaktivitätsunterschied von Diastereomeren hatte man auch bei der analogen Darstellung der 4-Methoxybicyclo[3.2.1] octa-2,6-diene beobachtet ${ }^{6 b}$. Bei terminal an den Vinylgruppen methylsubstituierten cis-Divinylcyclopropanen wurden die Einflüsse der Konfiguration auf die Geschwindigkeit der Umlagerung quantitativ erfa $\mathrm{t}^{32}$. Die Behandlung von 19a, b mit Kalium-tert-butoxid in Dimethylsulfoxid erbrachte das 2-Phenylisomere 20, in $\left[D_{6}\right]$ Dimethylsulfoxid das $\left[4,4-D_{2}\right]$-Derivat $\left[D_{2}\right]-20$.

Die Strukturen der Verbindungen $18 \mathrm{~b}$ und 19 stützen sich auf die NMR-Daten. Aufgrund der Größe der Kopplungskonstanten zwischen den Protonen der Styryldoppelbindung $\left(J_{1^{\prime}, 2^{\prime}}=11.8 \mathrm{~Hz}\right)$ steht die $Z$-Konfiguration fest. Zur Unterscheidung von $19 \mathrm{a}$ und $19 \mathrm{~b}$ dienen folgende Kriterien: $J_{4,5}$ beträgt 4.8 und $1.8 \mathrm{~Hz}$, was auf ähnliche Verhältnisse der Interplanarwinkel wie im Norbornansystem zurückgeht. Damit muß der größere Wert der endo-Phenylverbindung 19a zugeordnet werden. Der nach höherem Feld gerichtete Anisotropieeffekt der Phenylgruppe äußert sich wie erwartet bei der exo-Verbindung in den chemischen Verschiebungen der beiden $8-\mathrm{H}(19 \mathrm{~b}: \delta=1.69,1.86$ gegenüber 19a: $\delta=2.13$, 2.05), wobei interessanterweise $8-\mathrm{H}_{a n t i}$ stärker abgeschirmt wird als 8- $\mathrm{H}_{g y n}$, und bei der endo-Verbindung im Wert von 6-H (19a: $\delta=5.18$ gegenüber $19 \mathrm{~b}$ : $\delta=$ 5.93). In den ${ }^{13} \mathrm{C}-\mathrm{NMR}$-Spektren zeigt sich der $\gamma$-gauche-Effekt der Phenylgruppe in einer Hochfeldverschiebung des Signals von C-8 (19b: $\delta=35.76$ gegenüber 19a: $\delta=43.92)$ bzw. von C-6 (19a: $\delta=129.63$ gegenüber 19b: $\delta=131.53$ ).

Chem. Ber. 119 (1986) 
Tab. 1. ${ }^{1} \mathrm{H}$-NMR-Parameter von Bicyclo[3.2.1] octa-2,6-dienen in $\mathrm{CDCl}_{3}$ A. Chemische Verschiebungen ( $\delta$-Werte)

\begin{tabular}{|c|c|c|c|c|c|c|}
\hline Nr. & Substituent & $1-\mathrm{H}$ & $2-\mathrm{H}$ & 3-H & 4- $\mathrm{H}_{\text {endo }}$ & $4-\mathrm{H}_{\text {exo }}$ \\
\hline $\begin{array}{l}2 a) \\
4 \\
6 \\
16 \\
19 a \\
19 b \\
20\end{array}$ & 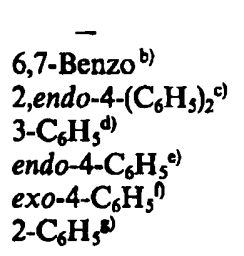 & $\begin{array}{r}\mathrm{m} 2.64 \\
\text { dd } 3.23 \\
\text { br. } \mathrm{t} 3.22 \\
\mathrm{~m} 2.89 \\
\mathrm{~m} 2.57 \\
\text { ddd } 2.70 \\
\mathrm{~m} 3.20\end{array}$ & $\begin{array}{c}\text { ddm } 6.06 \\
\text { br. dd } 6.12 \\
- \\
\text { br. d } 6.53 \\
\text { dddd } 6.24 \\
\text { dddd } 6.20 \\
-\end{array}$ & $\begin{array}{r}\operatorname{dm} 5.25 \\
\text { dm } 5.22 \\
\text { dt } 5.71 \\
- \\
\text { ddd } 5.33 \\
\text { ddd } 5.33 \\
\text { m } 5.60\end{array}$ & 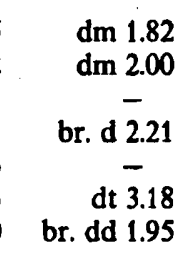 & $\begin{array}{r}\text { ddt } 2.33 \\
\text { ddt } 2.56 \\
\text { dd } 3.83 \\
\text { ddd } 2.68 \\
\text { dt } 3.62 \\
\overline{\text { ddd }} \overline{2.45}\end{array}$ \\
\hline Nr. & $5-\mathrm{H}$ & $6-\mathrm{H}$ & $7 \cdot \mathrm{H}$ & & $8-\mathrm{H}_{a n t i}$ & 8-H $\mathrm{H}_{s y n}$ \\
\hline $\begin{array}{l}2^{a)} \\
4 \\
6 \\
16 \\
19 a \\
19 b \\
20\end{array}$ & $\begin{array}{r}\mathrm{m} 2.70 \\
\mathrm{t} 3.28 \\
\mathrm{~m} 3.06 \\
\mathrm{~m} 2.92 \\
\text { tdd } 2.93 \\
\mathrm{~m} 2.56 \\
\mathrm{~m} 2.73\end{array}$ & $\begin{array}{l}\text { dd } 5.74 \\
-\overline{5 d} \\
\text { dd } 5.31 \\
\text { dd } 5.82 \\
\text { dd } 5.18 \\
\text { dd } 5.93 \\
\text { dd } 5.80\end{array}$ & $\begin{array}{l}\text { dd } 6.2 \\
- \\
\text { dd } 6.4 \\
\text { dd } 6.2 \\
\text { dd } 6.2 \\
\text { dd } 6.3 \\
\text { dd } 6.3\end{array}$ & br & $\begin{array}{r}\text { dt } 1.96 \\
\text { dt } 2.32 \\
\text { dt } 2.30 \\
\text { dt } 2.00 \\
\text { dtd } 2.13 \\
\text { dtd } 1.69 \\
\text { dt } 2.12\end{array}$ & $\begin{array}{l}\text { d } 1.74 \\
\text { d } 2.03 \\
\text { d } 2.19 \\
\text { d } 1.76 \\
\text { d } 2.05 \\
\text { d } 1.86 \\
\text { d } 1.85\end{array}$ \\
\hline
\end{tabular}

a) Zuordnungen durch Entkopplungsexperimente gesichert. - b) $7.03-7.11(\mathrm{~m}, 3 \mathrm{H}), 7.25$ (d, $J=7 \mathrm{~Hz}, 1 \mathrm{H})$ - c) 7.15, 7.43(o-H); 7.20, $7.26(p-\mathrm{H}) ; 7.26,7.33(\mathrm{~m}-\mathrm{H})$. d) 7.18(p-H), 7.27 $(m-\mathrm{H}), 7.34(0-\mathrm{H})$. $-\mathrm{e} 7.11(\mathrm{p}-\mathrm{H}), 7.24(0, \mathrm{~m}-\mathrm{H})$. $-7.05-7.25(\mathrm{~m}) . \mathrm{l}) 7.15-7.50(\mathrm{~m})$.

B. Kopplungskonstanten (in $\mathbf{H z}$ )

\begin{tabular}{|c|c|c|c|c|c|c|c|c|c|}
\hline $\mathrm{Nr}$ & $J_{1,2}$ & $J_{1,3}$ & $J_{1,7}$ & $J_{1, \text { Banti }}$ & $J_{2,3}$ & $J_{2,4 \text { endo }}$ & $J_{2 \text { texo }}$ & $J_{2,8 a n t i}$ & $J_{3, \text { endo }}$ \\
\hline $\begin{array}{l}2 \\
4\end{array}$ & $\begin{array}{l}6.3 \\
6.2\end{array}$ & $\begin{array}{l}0.8 \\
\text { a) }\end{array}$ & $\begin{array}{l}2.8 \\
-\end{array}$ & $\begin{array}{l}4.7 \\
4.8\end{array}$ & $\begin{array}{l}9.5 \\
9.5\end{array}$ & 2.4 & $\begin{array}{l}2.4 \\
2.5\end{array}$ & $\approx 1$ & ${ }_{21}^{2.6}$ \\
\hline 6 & - & 1.5 & 2.8 & 4.8 & - & - & - & - & - \\
\hline 16 & 6.5 & - & 2.8 & 4.6 & - & $\approx 1.5$ & 2.0 & a) & - \\
\hline $19 a$ & 6.3 & b) & 2.8 & 4.6 & 9.5 & - & 2.5 & 1.0 & - \\
\hline $19 b$ & 6.3 & b) & 2.8 & 4.6 & 9.7 & 1.8 & - & 1.0 & 3.1 \\
\hline 20 & - & a) & 2.7 & 4.8 & - & - & - & - & 4.2 \\
\hline Nr. & $J_{3, \text { Aexo }}$ & $\boldsymbol{J}_{3, \mathbf{S}}$ & $J_{4,4}$ & $J_{\text {Aend }, 5}$ & $J_{4 e \times 0,5}$ & $J_{5,6}$ & $J_{5,8 a n t i}$ & $J_{6,7}$ & $J_{8,8}$ \\
\hline 2 & 2.6 & 2.0 & 18.3 & 1.4 & 5.2 & 2.8 & 4.7 & 5.5 & 9.5 \\
\hline 4 & 2.5 & a) & 18.0 & a) & 4.8 & - & 4.8 & - & 9.8 \\
\hline 6 & 2.8 & 1.5 & - & - & 4.7 & 2.8 & 4.8 & 5.5 & 9.6 \\
\hline 16 & - & - & 18.0 & $\approx 1.5$ & 5.2 & 2.8 & 4.6 & 5.5 & 9.7 \\
\hline $19 a$ & 2.5 & 2.1 & - & - & 4.8 & 2.8 & 4.6 & 5.5 & 9.5 \\
\hline $19 b$ & - & 2.1 & - & 1.8 & - & 2.8 & 4.6 & 5.5 & 9.8 \\
\hline 20 & 3.3 & a) & 18.3 & a) & 5.0 & 2.7 & 4.8 & 5.5 & 9.6 \\
\hline
\end{tabular}

2) Wegen ungenügender Auflösung nicht ermittelt. $-{ }^{b},<0.9 \mathrm{~Hz}$.

Chem. Ber. 119 (1986) 
Tab. $2 .{ }^{13} \mathrm{C}-N M R$-Chemische Verschiebungen ( $\delta$-Werte), Multiplizitäten und Kopplungskonstanten über eine Bindung (in Hz, jeweils zweite Zeile) von Bicyclo[3.2.1] octa-2,6-dienen in $\mathrm{CDCl}_{3}$

\begin{tabular}{|c|c|c|c|c|c|c|c|c|c|}
\hline Nr. & Substituent & C-1 & $\mathrm{C}-2$ & C-3 & C-4 & C-5 & C-6 & C-7 & C-8 \\
\hline 2 & - & $\begin{array}{l}38.78^{a)} \\
\text { d } 145\end{array}$ & $\begin{array}{l}134.17^{b)} \\
d 161\end{array}$ & $\begin{array}{l}123.99^{b)} \\
\text { d } 160\end{array}$ & $\begin{array}{l}28.78 \\
\text { t } 127\end{array}$ & $\begin{array}{l}38.43^{a)} \\
\text { d } 141\end{array}$ & $\begin{array}{l}130.36^{b)} \\
d 168\end{array}$ & $\begin{array}{l}139.82^{b)} \\
\text { d } 169\end{array}$ & $\begin{array}{l}40.81 \\
\mathrm{t} 132\end{array}$ \\
\hline 4 & 6,7-Benzoc & $\begin{array}{l}40.70^{2)} \\
\text { d } 140\end{array}$ & $\begin{array}{l}134.19 \\
\text { d } 160\end{array}$ & $\begin{array}{l}123.48 \\
\text { d } 158\end{array}$ & $\begin{array}{l}32.30 \\
\text { t } 128\end{array}$ & $\begin{array}{l}40.31^{a)} \\
\text { d } 138\end{array}$ & $\begin{array}{l}146.25 \\
\text { s }\end{array}$ & $\begin{array}{l}151.90 \\
\mathrm{~s}\end{array}$ & $\begin{array}{l}41.25 \\
t 132\end{array}$ \\
\hline 6 & 2,endo-4-( $\left(\mathrm{C}_{6} \mathrm{H}_{5}\right)_{2}^{\text {d) }}$ & $\begin{array}{l}42.02 \\
\text { d } 142\end{array}$ & $l^{142.12^{d)}}$ & $\begin{array}{l}124.30 \\
\text { d } 158\end{array}$ & $\begin{array}{l}44.41 \\
\text { d } 128\end{array}$ & $\begin{array}{l}46.38 \\
\text { d } 142\end{array}$ & $\begin{array}{l}130.53 \\
\text { d } 168\end{array}$ & $\begin{array}{l}140.52 \\
\text { d } 165\end{array}$ & $\begin{array}{l}43.55 \\
t 130\end{array}$ \\
\hline 16 & $3-\mathrm{C}_{6} \mathrm{H}_{5}{ }^{8)}$ & $\begin{array}{l}38.93^{2)} \\
\text { d } 140\end{array}$ & $\begin{array}{l}129.73 \\
\text { d } 160\end{array}$ & $\begin{array}{l}133.16 \\
\mathrm{~s}\end{array}$ & $\begin{array}{l}30.03 \\
\text { t } 127\end{array}$ & $\begin{array}{l}38.31^{2)} \\
\text { d } 140\end{array}$ & $\begin{array}{l}130.94 \\
\text { d } 166\end{array}$ & $\begin{array}{l}139.07 \\
\text { d } 166\end{array}$ & $\begin{array}{l}40.82 \\
\mathrm{t} 132\end{array}$ \\
\hline 19a & endo-4- $\mathrm{C}_{6} \mathrm{H}_{3}{ }^{0}$ & $\begin{array}{l}38.49 \\
\text { d } 142\end{array}$ & $\begin{array}{l}134.53 \\
\text { d } 160\end{array}$ & $\begin{array}{l}126.15 \\
\text { d } 160\end{array}$ & $\begin{array}{l}44.47 \\
\text { d } 125\end{array}$ & $\begin{array}{l}46.89 \\
\text { d } 142\end{array}$ & $\begin{array}{l}129.63 \\
d 165\end{array}$ & $\begin{array}{l}141.14 \\
\text { d } 166\end{array}$ & $\begin{array}{l}43.92 \\
t 132\end{array}$ \\
\hline $19 b$ & $e x 0-4-\mathrm{C}_{6} \mathrm{H}_{5}^{2}$ & $\begin{array}{l}39.04 \\
\text { d } 142\end{array}$ & $\begin{array}{l}134.76 \\
\text { d } 160\end{array}$ & $\begin{array}{l}126.05 \\
d 160\end{array}$ & $\begin{array}{l}44.25 \\
\text { d } 125\end{array}$ & $\begin{array}{l}46.50 \\
\text { d } 142\end{array}$ & $\begin{array}{l}131.53 \\
\text { d } 165\end{array}$ & $\begin{array}{l}141.22 \\
\text { d } 166\end{array}$ & $\begin{array}{l}35.76 \\
t 132\end{array}$ \\
\hline
\end{tabular}

a) Zuordnung austauschbar. - " ${ }^{\circ}$ Zuordnung durch selektive 'H-Entkopplung gesichert. - o 120.26, 123.36 (jeweils dd, 158 Hz, 8 Hz; C-2',3'); 125.96, 126.03 (jeweils dt; $\left.160 \mathrm{~Hz}, 2 \mathrm{mal} 6.5 \mathrm{~Hz} ; \mathrm{C}^{1}, 4^{\prime}\right)$. - Unsere Zuordnung weicht bei einigen Positionen von der in Lit. ${ }^{\circ}$ ab. 124.79, 127.90

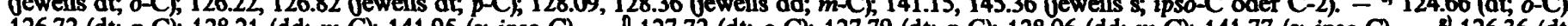
126.72 (dt; p-C); 128.21 (dd; m-C); 141.95 (s; ipso-C). - 127.72 (dt; o-C); 127.79 (dt; p-C); 128.06 (dd; m-C); 141.77 (s; ipso-C). - el 126.36 (dt; $p-C) ; 128.32$ (Signal mit 4facher Intensität, dt, dd; o,m-C); 145.01 (s; ipso-C). 
Zum Vergleich, insbesondere mit den Daten der zugehörigen Anionen, wurden in die Tab. 1 und 2 auch die zum Teil schon bekannten Parameter von 2, 4 und 6 aufgenommen. Die Literaturangaben ${ }^{9 c)}$ zum ${ }^{13} \mathrm{C}$-NMR-Spektrum von 6 enthalten einige Fehler. So sind die C-1 und C-4 zugeordneten Linien vertauscht, was jetzt aufgrund der ${ }^{13} \mathrm{C}-\mathrm{H}-\mathrm{Kopplungskonstanten} \mathrm{ans} \mathrm{Licht} \mathrm{kam.} \mathrm{Ebenso} \mathrm{wurde} \mathrm{die}$ zu C-3 gehörende Linie mit der von einem p-C-Atom einer Phenylgruppe verwechselt. Im protonengekoppelten Spektrum verursachen nämlich $p$-C-Atome wegen der Kopplung zu den $o$-H-Atomen eine Triplettfeinstruktur von ca. $8 \mathbf{H z}^{17 b)}$. Diese findet sich bei der $\delta=124.30$-Absorption im Gegensatz zu jenen bei 126.22 und 126.82 nicht, so daB erstere C-3 und die letzteren den p-C-Atomen der Phenylgruppen zugewiesen werden müssen. Weiterhin ist die Angabe für ${ }^{1} J_{\mathrm{C}, \mathrm{H}}$ von C-6 um $12 \mathrm{~Hz}$ zu klein.

Auch die Unterscheidung der Olefin- und Aromaten-C-Signale im ${ }^{13} \mathrm{C}-\mathrm{NMR}$ Spektrum von 4 gründet sich auf ${ }^{13} \mathrm{C}$-H-Fernkopplunen, die in Aromaten besonders konstante Werte aufweisen ${ }^{17 \mathrm{~b})}$. Während C-2,3 zu komplexeren Feinstrukturen Anlaß geben, erkennt man die Signale von $C-1^{\prime}, 2^{\prime}, 3^{\prime}, 4^{\prime}$ an zwei Doppeldubletts (je 158 und $8 \mathrm{~Hz}$ ) und zwei Doppeltripletts (je 160 und $2 \mathrm{mal} 6.5 \mathrm{~Hz}$ ). Wegen jeweils nur eines vicinalen Protons (4'-H bzw. 1'-H) müssen die Doppeldubletts $(\delta=120.26,123.26)$ von $C-2^{\prime}, 3^{\prime}$ stammen. $C-1^{\prime}, 4^{\prime}$ aber können mit je zwei vicinalen Protonen $\left(3^{\prime}-\mathrm{H}, 5-\mathrm{H}\right.$ bzw. $\left.2^{\prime}-\mathrm{H}, 1-\mathrm{H}\right)$ wechselwirken und sollten daher die Doppeltripletts verursachen.

\section{Erzeugung der Bicyclo[3.2.1]octadienyl-Anionen 5, 7, 21 und 22}

Trimitsis et al. ${ }^{9 b, c)}$ hatten 6 mit Butyllithium in Hexan zum Anion 7 deprotoniert. Es stellte sich jetzt heraus, daß die schwächere Base Methyllithium ausreicht.

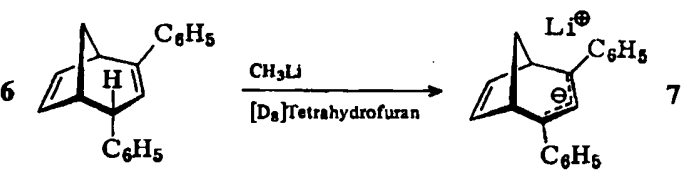

Zur Deprotonierung von 19a, b genügte die Basenstärke von Methyllithium nicht mehr, jedoch bildete sich mit Butyllithium glatt $21 \mathrm{Li}$. Sein ${ }^{13} \mathrm{C}-\mathrm{NMR}$-Spektrum zeigte aufgrund der Breite einiger Linien eine Temperaturabhängigkeit an, die durch Messung bei sechs Temperaturen zwischen -30 und $+45^{\circ} \mathrm{C}$ untersucht wurde. In Tab. 4 sind nur die Ergebnisse bei den extremen Temperaturen angegeben. Das ${ }^{1} \mathrm{H}$-NMR-Spektrum wurde bei zwei Temperaturen aufgenommen, wobei einige Fremdbanden die Signale von $8-\mathrm{H}_{2}$ überlagern.

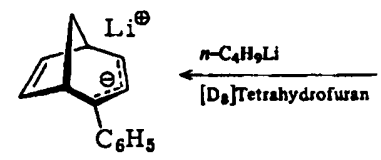

$21 \mathrm{Li}$

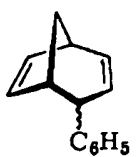

19 a, b

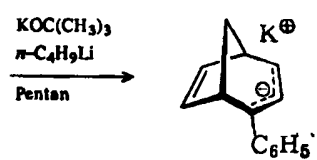

$21 \mathrm{~K}$

Chem. Ber. 119 (1986) 
Um den Einfluß des Gegenions auf die NMR-Absorptionen des Anions $21 \mathrm{zu}$ studieren, wollten wir auch $21 \mathrm{~K}$ darstellen. Die Chance dazu bestand, nachdem es Köhler und Hertkorn ${ }^{20)}$ gelungen war, 2 mit Hilfe der sog. Schlosser-Base ${ }^{33)}$, einer Mischung aus Kalium-tert-butoxid und $n$-Butyllithium, zu deprotonieren. Frühere Versuche zur Umwandlung von 2 in 1 mit Isopropyllithium, Cumylkalium und Triphenylmethyllithium waren gescheitert ${ }^{6 a}$. Die stärkere Base tert-Butyllithium abstrahiert überraschend ein Proton der Positionen 6 oder 7 von $2^{34}$. DaB 2 mit Schlosser-Base in 1 mit $\mathrm{K}^{+}$als Gegenion übergeht, beweisen die ${ }^{13} \mathrm{C}$-chemischen Verschiebungen ${ }^{20}$, die genau gleich denen von authentischem 1 mit $\mathrm{K}^{+}$ als Gegenion ${ }^{21}$ sind.

Entsprechend der Vorschrift für $2 \rightarrow 1^{201}$ behandelten wir 19a, b mit Kalium-tertbutoxid/ $n$-Butyllithium in Pentan und lösten den dabei gebildeten Niederschlag zur Spektroskopie in $\left[\mathrm{D}_{8}\right]$ Tetrahydrofuran. Im ${ }^{1} \mathrm{H}$-NMR-Spektrum überlagern intensive Multipletts bei $\delta=0.8-1.6$ die 8- $\mathrm{H}_{2}$-Signale, jedoch stören die Verunreinigungen im ${ }^{13} \mathrm{C}-\mathrm{NMR}$-Spektrum nicht. Letzteres erwies sich im Gegensatz zu dem von $21 \mathrm{Li}$ im Bereich von $20-45^{\circ} \mathrm{C}$ als nicht temperaturabhängig. Dieser Befund sowie die deutlichen Unterschiede der chemischen Verschiebungen im Vergleich zu denen von $21 \mathrm{Li}$, besonders im ${ }^{13} \mathrm{C}$-NMR-Spektrum, lassen an der Bildung von $21 \mathrm{~K}$ keinen $Z$ weifel.

Die Kohlenwasserstoffe 4 und 16 wurden analog mit Schlosser-Base in die Anionen 5 bzw. 22 umgewandelt. Ihre NMR-Proben enthielten die schon bei 21K beobachteten Verunreinigungen und waren auch bei tiefer Temperatur nur kurzzeitig haltbar. Die Ursache der Labilität ist bei 5 die starke Basizität. Das Auftauchen der Signale von in 4-Stellung monodeuteriertem 4 spricht für die Abstraktion eines Deuterons vom Lösungsmittel $\left[\mathrm{D}_{8}\right]$ Tetrahydrofuran. Bei 22 kennen wir den Grund für die Zersetzung nicht, die sich in einer Zunahme der Viskosität äußerte und in den NMR-Spektren breite Banden auch im Bereich der Signale olefinischer $\mathrm{H}$ - und $\mathrm{C}$-Atome veranlaßte.
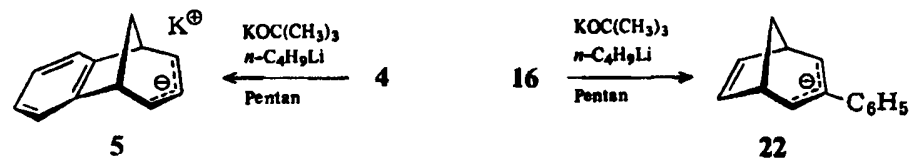

Die Tab. 3 und 4 fassen die ${ }^{1} \mathrm{H}$ - bzw. ${ }^{13} \mathrm{C}-\mathrm{NMR}$-Parameter aller Anionen zusammen. Im Vergleich mit den zugehörigen Kohlenwasserstoffen (Tab. 1 und 2) beseitigt die höhere Symmetrie einige Zuordnungsprobleme. Beim unsymmetrischen $21 \mathrm{Li}$ sichern Entkopplungsexperimente im ${ }^{1} \mathrm{H}$-NMR-Spektrum die Charakterisierung aller Signale. Nahezu ausnahmslos beugen die typischen Multiplizitäten einer Verwechslung von ${ }^{1} \mathrm{H}$-Absorptionen vor. Bei den ${ }^{13} \mathrm{C}$-NMR-Spektren stützen sich die Zuweisungen in erster Linie auf die ${ }^{13} \mathrm{C}$-H-Kopplungskonstanten über eine Bindung. Allerdings war die Auflösung nicht gut genug, um Feinstrukturen aufgrund weitreichender ${ }^{13} \mathrm{C}$-H-Kopplungskonstanten heranziehen zu können, was besonders für die Zuordnung der Banden aromatischer C-Atome von Bedeutung gewesen wäre. Als problematisch mag die Interpretation der Spektren

Chem. Ber. 119 (1986) 
Tab. 3. ${ }^{1} \mathrm{H}$-NMR-Chemische Verschiebungen ( $\delta$-Werte) und Kopplungskonstanten von Bicyclo[3.2.1] ]octa-3,6-dien-2-yl-Anionen in $\left[D_{8}\right]$ Tetrahydrofuran, interner Standard $\left[D_{8}\right]$ Tetrahydrofuran-Signal bei $\delta=3.58^{17 c}$

\begin{tabular}{|c|c|c|c|c|c|c|c|c|c|c|c|c|}
\hline Nr. & $\begin{array}{c}\text { Substi- } \\
\text { tuent }\end{array}$ & $\begin{array}{l}\text { Ge- } \\
\text { gen- } \\
\text { ion }\end{array}$ & $\underset{\left({ }^{\circ} \mathrm{C}\right)}{\text { Temp. }}$ & $1,5-\mathrm{H}$ & 2,4-H & $3-\mathrm{H}$ & $6,7-\mathrm{H}$ & $8-\mathrm{H}_{\text {anct }}$ & $8-\mathrm{H}_{3 \mathrm{n}}$ & $0-\mathbf{H}$ & $m-\mathbf{H}$ & $p-\mathbf{H}$ \\
\hline 1) & - & $\mathbf{K}^{+}$ & 25 & m 2.46 & m 2.84 & tt 5.40 & br. s 3.64 & ddt 0.90 & dqui 0.43 & - & - & - \\
\hline $5^{b /}$ & 6,7-Benzo & $\mathbf{K}^{+}$ & -50 & m 3.16 & $\mathrm{~m} 2.58$ & t 5.42 & - & c) & d & $\begin{array}{l}1^{\prime}, 4^{\prime}-\mathrm{H}: \\
6.72^{d)}\end{array}$ & m $6.88^{d)}$ & $2^{\prime}, 3^{\prime}-\mathrm{H}: \mathrm{m}$ \\
\hline$\gamma^{t}$ & $2,4-\left(\mathrm{C}_{6} \mathrm{H}_{5}\right)_{2}$ & $\mathrm{Li}^{+}$ & 25 & br. d 3.33 & - & br. s 6.71 & br. s 4.83 & dt 1.63 & d 1.35 & $6.88^{n}$ & t $6.67^{\mathrm{g})}$ & t $5.97^{(\mathrm{h})}$ \\
\hline \multirow[t]{2}{*}{$21 \mathrm{Li}^{\mathrm{i})}$} & $2-\mathrm{C}_{6} \mathrm{H}_{5}$ & $\mathbf{L i}^{+}$ & 25 & m 2.35' & dd 3.73 & br. d 5.81 & dd 4.11") & c) & c) & m 6.31 & m 6.31 & m 5.35 \\
\hline & & & -30 & $\begin{array}{l}2.33^{\mathrm{i})} \\
3.13^{\mathrm{k})}\end{array}$ & 3.75 & 5.75 & $\begin{array}{l}4.08^{(3)} \\
4.54^{\mathrm{m})}\end{array}$ & c) & c) & m 6.22 & m 6.22 & m 5.23 \\
\hline $21 \mathrm{~K}$ & $2-\mathrm{C}_{6} \mathrm{H}_{5}$ & $\mathbf{K}^{+}$ & 25 & $\begin{array}{l}2.50^{\mathrm{j}} \\
3.31^{\mathrm{k})}\end{array}$ & 4.04 & 5.89 & $\begin{array}{l}4.32^{\prime \prime} \\
4.79^{\mathrm{mm}}\end{array}$ & c) & $\Rightarrow$ & $6.44^{n}$ & $6.51^{81}$ & $t 5.57^{\mathrm{b})}$ \\
\hline $22^{\mathrm{a})}$ & $3-\mathrm{C}_{6} \mathrm{H}_{5}$ & $\mathbf{K}^{+}$ & -50 & m $\left.2.70^{\circ}\right)$ & 3.28 & - & br. s 3.77 & c) & d 0.55 & $7.51^{n}$ & 7.08 & 6.94 \\
\hline \multicolumn{13}{|c|}{ 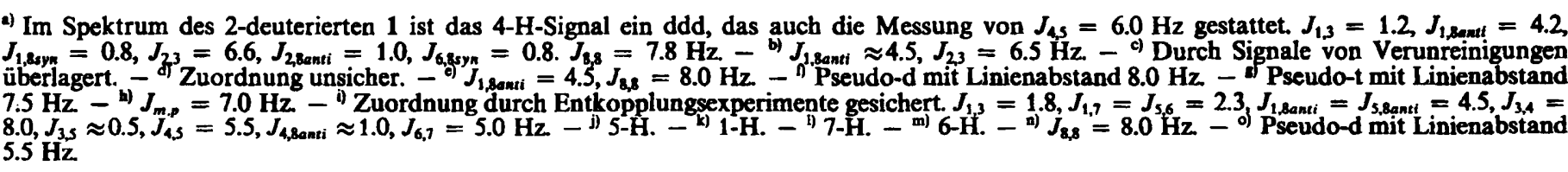 } \\
\hline
\end{tabular}




\begin{tabular}{|c|c|c|c|c|c|c|c|c|c|c|c|c|}
\hline Nr. & $\begin{array}{c}\text { Substi- } \\
\text { tuent }\end{array}$ & $\begin{array}{c}\text { Gegen- } \\
\text { ion }\end{array}$ & $\begin{array}{l}\text { Temp. } \\
\left({ }^{\circ} \mathrm{C}\right)\end{array}$ & $C-1,5$ & $C-2,4$ & C-3 & $C-6,7$ & C-8 & ipso-C & $0-\mathrm{C}$ & $m-\mathrm{C}$ & $p-C$ \\
\hline 1 & - & $\mathbf{K}^{+}$ & 45 & $\begin{array}{l}40.10 \\
\text { d } 135\end{array}$ & $\begin{array}{l}58.31 \\
\text { d } 157\end{array}$ & $\begin{array}{l}120.02 \\
\text { d } 140\end{array}$ & $\begin{array}{l}91.02 \\
d 155\end{array}$ & $\begin{array}{l}31.74 \\
t 130\end{array}$ & - & - & - & - \\
\hline 5 & 6,7-Benzo & $\mathbf{K}^{+}$ & -50 & $\begin{array}{l}43.55 \\
\text { d } 135\end{array}$ & $\begin{array}{l}62.18 \\
\text { d } 161\end{array}$ & $\begin{array}{l}122.59 \\
\text { d } 139\end{array}$ & $\begin{array}{l}129.32 \\
\mathrm{~s}\end{array}$ & $\begin{array}{r}32.39 \\
\mathrm{t} 132\end{array}$ & C-1', $4^{\prime}:$ & $\begin{array}{l}122.86^{2)} \\
\text { d } 158\end{array}$ & $C-2^{\prime}, 3^{\prime}:$ & $\begin{array}{l}119.34^{2)} \\
\text { d } 156\end{array}$ \\
\hline 7b) & $2,4-\left(C_{6} H_{5}\right)_{2}$ & $\mathbf{L i}^{+}$ & 45 & $\begin{array}{l}41.74 \\
d\end{array}$ & $\begin{array}{l}94.28 \\
\mathrm{~s}\end{array}$ & ${ }_{d}^{115.77}$ & ${ }_{d}^{117.95}$ & $\begin{array}{l}37.55 \\
t\end{array}$ & 144.96 & $\begin{array}{l}117.27 \\
d\end{array}$ & ${ }_{d}^{128.15}$ & $\mathrm{~d}^{111.73}$ \\
\hline \multirow[t]{2}{*}{$21 \mathrm{Li}$} & $2-\mathrm{C}_{6} \mathrm{H}_{5}$ & $\mathrm{Li}^{+}$ & 45 & $\begin{array}{l}40.16 \\
40.64\end{array}$ & $\begin{array}{l}\approx 79.0 \\
\approx 79.0\end{array}$ & 116.37 & $\begin{array}{l}107.55 \\
108.60\end{array}$ & 35.27 & 144.23 & 114.68 & 127.91 & 107.55 \\
\hline & & & -30 & $\begin{array}{l}40.77 \\
\text { d 134 } \\
40.77 \\
\text { d } 134\end{array}$ & $\begin{array}{l}\widetilde{83.40} \\
\text { d } 158 \\
80.62 \\
\text { s }\end{array}$ & $\begin{array}{l}118.35 \\
\text { d } 141\end{array}$ & $\begin{array}{l}110.10 \\
d 158 \\
111.64 \\
d 158\end{array}$ & $\begin{array}{l}36.19 \\
\text { t } 130\end{array}$ & $\begin{array}{l}143.27 \\
\mathrm{~s}\end{array}$ & $\begin{array}{l}112.00 \\
d \quad 150 \\
113.07 \\
d \text { d } 149\end{array}$ & $\begin{array}{l}127.49 \\
\text { d 148 } \\
128.25 \\
\text { d } 147\end{array}$ & $\begin{array}{l}103.85 \\
d 157\end{array}$ \\
\hline $21 K$ & $2-\mathrm{C}_{6} \mathrm{H}_{5}$ & $\mathbf{K}^{+}$ & 45 & $\begin{array}{l}40.73 \\
d 138 \\
40.90 \\
d 138\end{array}$ & $\begin{array}{l}86.13 \\
\text { d } 160 \\
79.04 \\
s\end{array}$ & $\begin{array}{l}\mathbf{1 1 7 . 5 2} \\
\mathrm{d} 142\end{array}$ & $\begin{array}{l}111.10 \\
\text { d } 160 \\
112.96 \\
\text { d } 160\end{array}$ & $\begin{array}{l}36.52 \\
t 130\end{array}$ & $\begin{array}{l}143.37 \\
s\end{array}$ & 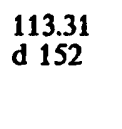 & $\begin{array}{l}129.61 \\
\text { d } 148\end{array}$ & $\begin{array}{l}106.86 \\
\text { d } 158\end{array}$ \\
\hline 22 & $3-\mathrm{C}_{6} \mathrm{H}_{5}$ & $\mathbf{K}^{+}$ & -50 & $\begin{array}{l}39.72 \\
\text { d } 138\end{array}$ & $\begin{array}{l}56.73 \\
\text { d } 159\end{array}$ & $\begin{array}{l}128.65 \\
\mathrm{~s}\end{array}$ & $\begin{array}{l}92.71 \\
\text { d } 157\end{array}$ & $\begin{array}{l}32.13 \\
t 129\end{array}$ & $\begin{array}{l}147.72 \\
\mathrm{~s}\end{array}$ & $\begin{array}{l}125.22^{2)} \\
\text { d } 160\end{array}$ & $\begin{array}{l}128.04^{2)} \\
d 156\end{array}$ & $\begin{array}{l}124.47 \\
\text { d } 159\end{array}$ \\
\hline
\end{tabular}


von $21 \mathrm{Li}$ und $21 \mathrm{~K}$ erscheinen, da im Absorptionsbereich olefinischer und aromatischer C-Atome eine große Liniendichte auftritt. Jedoch sind im Spektrum von $21 \mathrm{Li}$ bei $45^{\circ} \mathrm{C}$ die $o$ - und $m$-C-Signale der Phenylgruppe an der doppelten Intensität erkennbar. Wenn auch die $p$-C-Linie bei dieser Temperatur mit einer der C-6,7-Signale zusammenfällt, so schließt doch die Wanderungsrichtung dieser Linien beim Abkühlen der Probe eine Fehlzuweisung aus. Naturgemä $B$ wäre eine Unterscheidung innerhalb der Signalpaare von C-1,5, C-6,7, o, $o^{\prime}-\mathrm{C}$ und $m, m^{\prime}-\mathrm{C}$ aufwendig und auch nicht von großem Nutzen.

\section{Diskussion}

Die Tab. 5 enthält die Effekte der Deprotonierung auf die ${ }^{13} \mathrm{C}$-chemischen Verschiebungen der Kohlenwasserstoffe 2, 4, 6, 16, 19a und 19b, und zwar für die einzelnen C-Atom-Typen und, in der letzten Spalte, auch die Summe über alle C-Atome als Differenz zwischen den chemischen Verschiebungen eines Bicyclo[3.2.1]octa-2,6-diens und des zugehörigen Bicyclo[3.2.1]octadienyl-Anions. Die Deprotonierungseffekte auf die ${ }^{1} \mathrm{H}$-chemischen Verschiebungen zeigen im wesentlichen das gleiche Bild wie jene auf die ${ }^{13} \mathrm{C}$-chemischen Verschiebungen und werden daher nicht eigens diskutiert.

Wie schon in Abschnitt A erwähnt, haben wir den Maximalwert des Effektes von -88.14 ppm auf C-6,7 bei $2 \rightarrow 1$ auf die Ladungsaufnahme durch diese CAtome und die Erhöhung ihrer Koordinationszahl (Umhybridisierung in Richtung $\mathrm{sp}^{3}$ ) infolge der homokonjugativen Wechselwirkung mit C-2,4 zurückgeführt. Der zweitgrößte Effekt tritt mit $-46.33 \mathrm{ppm}$ bei C-2,4 auf und enthält die Wirkung der Umhybridisierung von $\mathrm{C}-4$ von $\mathrm{sp}^{3}$ nach $\mathrm{sp}^{2}$. $\mathrm{DaB}$ die chemische Verschiebung von $C-2,4$ in 1 mit $\delta=58.31$ recht ähnlich derjenigen von $C-1,3$ von Allylkalium $\left(23, \delta=52.8^{19}\right)$ ist, liegt wohl auch an der Homokonjugation mit C-6,7, denn das von 3 abgeleitete Allyl-Anion, dessen NMR-Spektren leider bisher nicht verfügbar sind, dürfte aufgrund der chemischen Verschiebungen des Isopropylallyl-Anions ${ }^{196)}$ einen Wert bei erheblich niedrigerem Feld aufweisen.

Die Summe der Deprotonierungseffekte bei $2 \rightarrow 1$ von $-144.52 \mathrm{ppm}$ in Tab. 5 geht durch Benzoanellierung bei $4 \rightarrow 5$ auf $-96.51 \mathrm{ppm}$ zurück. Den Hauptbeitrag dieser Differenz verursacht der Benzolkern, dessen sechs C-Atome nur eine Gesamtwirkung von $-50.72 \mathrm{ppm}$ erfahren. Zusammen mit dem ebenfalls verringerten C-2,4-Wert $(-42.13$ gegenüber -46.33 bei $2 \rightarrow 1$ ) spricht dies für einen weniger ausgeprägten bishomoaromatischen Charakter von 5. Damit im Einklang steht die im Vergleich zu 2 verringerte Acidität von $4^{8}$ (vgl. Abschnitt A) und die im Vergleich zu 1 erhöhte Basizität von 5 (vgl. Abschnitt B. 3). Schon ein einfaches MO-Bild kann den Unterschied von 1 und 5 deuten. Die MO-Methode beschreibt die homokonjugative Wechselwirkung in 1 als Mischung des HOMOs des AllylAnion-Teils $\left(\varphi_{2}\right)$ mit dem LUMO der Vinylenbrücke ( $\pi^{*}$, vgl. Abb. 2). Das neue MO, das hauptsächlich $\varphi_{2}$-Charakter aufweist, erfährt dabei die entscheidende Stabilisierung.

Nach HMO sollte die Phenylenbrücke von 5 als Elektronenakzeptor weniger geeignet sein als die Vinylenbrücke von 1. Zwar weist das als LUMO dienende

Chem. Ber. 119 (1986) 


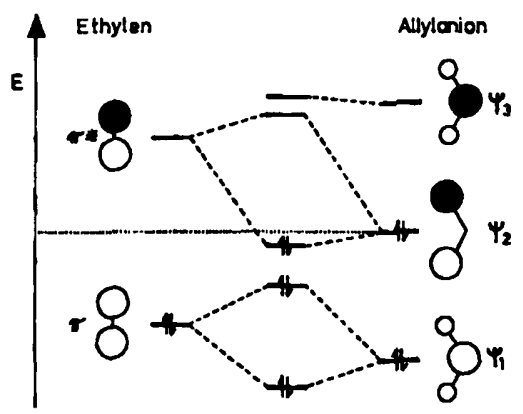

Abb. 2. MO-Schema zur homokonjugativen Wechselwirkung im Bicyclo[3.2.1]octadienylAnion 1

Benzol- $e_{2 \mathrm{u}}$-Orbital die gleiche Energie auf wie das Ethylen- $\pi^{*}$-Niveau $(-1.00 \beta)$, jedoch betragen die Koeffizienten von Belang nur 0.500 gegenüber $0.707^{35}$.

Da die 4-Phenylbicyclo[3.2.1] octadiene 19a, b schon durch $n$-Butyllithium glatt deprotoniert werden, sind sie erheblich acider als der unsubstituierte Kohlenwasserstoff 2, was sich auf eine Stabilisierung des Anions 21 relativ zu 1 gründet. Wie die chemischen Verschiebungen dartun, erniedrigt die Phenylgruppe die Energie des Allyl-Anion-Systems durch Ubernahme von negativer Ladung, was in der MO-Sprache als Mischung des $\varphi_{2}$-Orbitals mit dem Benzol- $e_{2 u} z u$ beschreiben ist. Dies äußert sich in einer massiven Abschirmung der $o$ - und $p$-C-Atome $(21 \mathrm{Li}$ bei $45^{\circ} \mathrm{C}: \delta=114.68$ bzw. 107.55$)$ im Vergleich zu jenen von 19a,b $(\delta=127.72$, 128.32 bzw. 127.79, 126.36). Die Mesomerie mit der Phenylgruppe tritt in Konkurrenz zur homokonjugativen Wechselwirkung $C-2,4 / C-6,7$ und schwächt diese im Vergleich zu der in $1 \mathrm{ab}$, was sich aus den Zahlen der Tab. 5 klar ablesen läßt. Die Deprotonierungseffekte auf C-2,4 und C-6,7 sind auf ca. 45 bzw. $63 \%$ der Werte von $\mathbf{2} \rightarrow \mathbf{1}$ gefallen.

Beim Abkühlen von $21 \mathrm{Li}$ verstärkt sich die geschilderte Tendenz, weil die bei $45^{\circ} \mathrm{C}$ nicht eingeschränkte Rotation um die C-2/ipso-C-Bindung schon bei $-30^{\circ} \mathrm{C}$ einfriert und sich so bessere Mesomeriebedingungen ergeben. Vier PhenylC-Signale im Spektrum bei $45^{\circ} \mathrm{C}$ und deren sechs in jenem bei $-30^{\circ} \mathrm{C}$ beweisen diesen Sachverhalt. Die koplanare Anordnung von Allyl- und Phenyleinheit folgt zwanglos aus der weiteren Abschirmung von $o$ - und $p-C(\delta=112.0,113.07$ bzw. 103.85) und der gleichzeitigen Entschirmung von C-2,4 und C-6,7. Die Deprotonierungseffekte (Tab. 5) auf die letzteren betragen jetzt nur noch ca. 32 bzw. 57\% der Werte von 1 . Im Gegensatz zu dieser Temperaturabhängigkeit waren beim Abkühlen von 1 kleine Hochfeldverschiebungen der Signale von C2,4 und C-6,7 beobachtet worden ${ }^{21)}$.

Eine weitere Schwächung der Homokonjugation führt der Austausch des Gegenions $\mathrm{Li}^{+}$gegen $\mathrm{K}^{+}$herbei. Dies spiegeln sowohl die Deprotonierungseffekte auf C-2,4 und C-6,7 mit ca. 30 bzw. 54\% der Werte von $2 \rightarrow 1$ als auch der Gesamteffekt in der letzten Spalte von Tab. 5 wider. Der Vergleich der bei $45^{\circ} \mathrm{C}$ gemessenen Spektren von $21 \mathrm{Li}$ und $21 \mathrm{~K}$ offenbart indessen, daß sich die chemischen Verschiebungen der Phenyl-C-Atome nur geringfügig unterscheiden. Allerdings friert bei $21 \mathrm{~K}$ die Rotation um die C-2/ipso-C-Bindung bei $-30^{\circ} \mathrm{C}$ noch

Chem. Ber. 119 (1986) 
Tab. 5. Effekte der Deprotonierung von Bicyclo[3.2.1]octa-2,6-dienen zu Bicyclo[3.2.1]octa-3,6-dien-2-yl-Anionen auf die ${ }^{13} \mathrm{C}-\mathrm{NMR}$-chemischen Verschiebungen (in ppm)

\begin{tabular}{|c|c|c|c|c|c|c|c|c|c|c|}
\hline $\begin{array}{l}\text { Verbindungs- } \\
\text { paar }\end{array}$ & $C-1,5^{b)}$ & $C-2,4^{b)}$ & C-3 & $C-6,7^{\text {b) }}$ & C-8 & $i p s a-C$ & $a-C^{b)}$ & $m-C^{b)}$ & $p-C$ & Summe \\
\hline $2 \rightarrow 1$ & +2.99 & -46.33 & -3.97 & -88.14 & -9.07 & - & - & - & - & -144.52 \\
\hline $4 \rightarrow 5$ & +6.09 & -42.13 & -0.89 & $-50.72^{c}$ & -8.86 & - & - & - & - & -96.51 \\
\hline $6 \rightarrow 7$ & -4.92 & $+2.03^{d)}$ & -8.53 & -35.15 & -6.00 & $+3.41^{b)}$ & $-36.30^{\varepsilon)}$ & $-0.30^{e)}$ & $-29.58^{b)}$ & $-115.34^{d)}$ \\
\hline $19 a \rightarrow 21 L^{* n}$ & $\begin{array}{l}-4.58 \\
-3.84\end{array}$ & $\begin{array}{l}-21.00 \\
-14.98\end{array}$ & $\begin{array}{l}-9.78 \\
-7.80\end{array}$ & $\begin{array}{l}-54.62 \\
-49.03\end{array}$ & $\begin{array}{l}-8.65 \\
-7.73\end{array}$ & $\begin{array}{l}+2.46 \\
+1.50\end{array}$ & $\begin{array}{l}-26.08 \\
-30.37\end{array}$ & $\begin{array}{l}-0.30 \\
-0.38\end{array}$ & $\begin{array}{l}-20.24 \\
-23.94\end{array}$ & $\begin{array}{l}-142.79 \\
-136.57\end{array}$ \\
\hline $19 \mathrm{~b} \rightarrow 21 \mathrm{~L}^{\mathrm{n}}$ & $\begin{array}{l}-4.74 \\
-4.00\end{array}$ & $\begin{array}{l}-21.01 \\
-14.99\end{array}$ & $\begin{array}{l}-9.68 \\
-7.70\end{array}$ & $\begin{array}{l}-56.60 \\
-51.01\end{array}$ & $\begin{array}{l}-0.49 \\
+0.43\end{array}$ & $\begin{array}{l}-0.78 \\
-1.74\end{array}$ & $\begin{array}{l}-27.28 \\
-31.57\end{array}$ & $\begin{array}{l}-0.82 \\
-0.90\end{array}$ & $\begin{array}{l}-18.81 \\
-22.51\end{array}$ & $\begin{array}{l}-140.21 \\
-134.09\end{array}$ \\
\hline $19 a \rightarrow 21 K$ & -3.75 & -13.83 & -8.63 & -46.71 & -7.40 & +1.60 & -28.82 & +3.10 & -20.93 & -125.37 \\
\hline $19 b \rightarrow 21 K$ & -3.91 & -13.84 & -8.53 & -48.69 & +0.76 & -1.64 & -30.02 & +2.58 & -19.50 & -122.07 \\
\hline $16 \rightarrow 22$ & +2.20 & -46.30 & -4.51 & -84.59 & -8.69 & +5.77 & +1.12 & -0.34 & -2.25 & -137.59 \\
\hline
\end{tabular}

a) Negative Vorzeichen entsprechen einer Veränderung nach höherem Feld. - ") Summe der Effekte auf zwei C-Atome. - ") Summe der Effekte auf die sechs Aromaten-C-Atome. - d) Wegen unsicherer Zuordnung Abweichung um einige ppm möglich. - e) Summe der Effekte auf vier

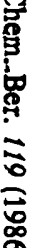


nicht ein. $\mathrm{K}^{+}$als Gegenion schränkt also besagte Drehbarkeit weniger ein als $\mathrm{Li}^{+}$. Zwar erbringt die Koplanarität von Allyl- und Phenyleinheit eine elektronische Stabilisierung aufgrund verbesserter Mesomerie, muß aber erhöhte sterische Spannung wegen der AbstoBung zwischen 1-H und 3-H einerseits und den beiden o-H-Atomen andererseits (Analogie zu Biphenyl) in Kauf nehmen. Im Falle von $21 \mathrm{~K}$ spielt also der weitere Energiegewinn durch optimierte Mesomerie eine geringere Rolle als bei $21 \mathrm{Li}$. Augenscheinlich stabilisiert das weiche Kation $\mathrm{K}^{+}$das weiche Allyl-Anion-System besser, als dies das harte Kation $\mathrm{Li}^{+}$kann.

Unterschiedliche NMR-Spektren von Allyl-Anionen als Folge unterschiedlicher Gegenionen sind nicht neu. So weichen die chemischen Verschiebungen von Allylkalium (23) und Allyllithium $\left(\delta=51.1,147.0^{36}\right)$ deutlich voneinander ab. Noch größer ist die Differenz zwischen den Werten von Phenylallyllithium $24 \mathbf{L i}^{36)}$ und Phenylallylkalium $24 \mathrm{~K}^{19 a}$. $\mathrm{DaB}$ die Änderungen beim Úbergang $24 \mathrm{Li} \rightarrow 24 \mathrm{~K}$ weder hinsichtlich der Größe noch der Vorzeichen als Vorbild für die bei $21 \mathrm{Li} \rightarrow 21 \mathrm{~K}$ $\left(45^{\circ} \mathrm{C}\right)$ dienen können, ist eine weitere Manifestation einer zusätzlichen Wechselwirkung, nämlich der homokonjugativen.
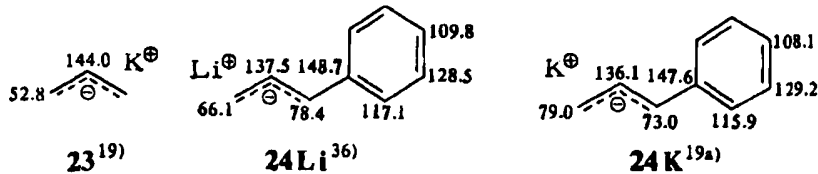

Das System 21 gewährt somit einen umfassenden Einblick in die Stabilisierungsmöglichkeiten des Bicyclo[3.2.1]octadienyl-Anions, die alle darauf hinauslaufen, die negative Ladung möglichst breit zu verteilen. Trotz des starken Einflusses der Phenylgruppe bleibt aber ein Teil der homokonjugativen Wechselwirkung erhalten.

2,4-Diphenylbicyclo[3.2.1] octadien (6) gibt das Allylproton noch leichter ab als die Monophenylverbindungen 19. Schon Methyllithium reicht aus, um das Anion 7 quantitativ zu erzeugen. Also muß die Einführung einer zweiten Phenylgruppe in $21 \mathrm{am}$ anderen Ende des Allyl-Anion-Systems dieses weiter stabilisieren. Zwar signalisieren die chemischen Verschiebungen von $o$ - und $p-C(\delta=117.27 \mathrm{bzw}$. 111.73) weniger negative Ladung pro Phenylgruppe als in 21, aber die Gesamtwirkung beider Substituenten scheint im Vergleich zu 21 um ca. 50\% gesteigert zu sein, wenn man den Deprotonierungseffekt auf $p-C$ in Tab. 5 als Maß nimmt. Dies geht auf Kosten der homokonjugativen Wechselwirkung. Bei C-2,4 ist der Deprotonierungseffekt mit $+2.03 \mathrm{ppm}$ schon entschirmend, wohl weil jetzt der Einfluß der Umhybridisierung von $\mathrm{C}-4$ von $\mathrm{sp}^{3}$ nach $\mathrm{sp}^{2}$ die Oberhand gewinnt, jedoch ergibt sich der C-6,7-Wert mit $-35.15 \mathrm{ppm}$ immer noch zu $40 \%$ desjenigen von $2 \rightarrow 1$. Darin zeigt sich nach wie vor, wenn auch weiter abgeschwächt, aber immer noch aufrechterhalten, die Homokonjugation C-2,4/C-6,7. Die chemischen Verschiebungen der Phenyl-C-Atome von 7 sind denen von 1,3-Diphenylallylnatrium ${ }^{3 n}$ sehr ähnlich.

Leider können die Deprotonierungseffekte der Tab. 5 nicht in Stabilisierungsenergien der Anionen umgerechnet werden. Aber durch Arbeiten von Saunders et $a{ }^{38)}$ wei $B$ man, $d a B$ minimale Energieänderungen große Wirkungen auf die che-

Chem. Ber. 119 (1986) 
mischen Verschiebungen haben können. Damit sei betont, daß die nichtklassische Stabilisierung in 7 durchaus sehr klein sein könnte, d. h. kleiner als $1 \mathrm{kcal} / \mathrm{mol}$, aber ihre Existenz durch die ${ }^{13} \mathrm{C}$-chemischen Verschiebungen von C-6,7 noch deutlich nachgewiesen wird.

Mit der Untersuchung des 3-Phenylbicyclo[3.2.1]octadienyl-Anions (22) wollten wir prüfen, ob die Vinylenbrücke (C-6,7) die negative Ladung teilweise an das Allylsystem zurūckgibt. In der MO-Sprache lautet die Frage, ob in 1 über die Wechselwirkung $\varphi_{2}-\pi^{*}$ hinaus auch die zwischen $\pi$ und $\varphi_{3}$ (vgl. Abb. 2) eine Rolle spielt. Die Valenzstrich-Schreibweise drückt dies durch die tetracyclische Grenzformel in Abb. 1 (rechts) aus. Die ins Allylsystem zurückfließende Ladung würde zumindest teilweise an C-3 von 1 auftauchen und müBte daher in 22 durch den mesomeren Effekt der Phenylgruppe stabilisiert werden. DaB dies nicht der Fall ist, folgt eindeutig aus dem ${ }^{13} \mathrm{C}$-NMR-Spektrum. Zwar ergibt sich für die chemische Verschiebung des Phenyl-p-C von 22 im Vergleich zu 16 ein Hochfeldeffekt von $2.25 \mathrm{ppm}$ (Tab. 5), aber dieser resultiert aus der Übernahme einer kleinen Partialladung vom Allyl-Anion-System auf induktivem Wege ${ }^{39}$. Die Begründung dafür leitet sich aus den Deprotonierungseffekten auf C-2,4 und C-6,7 ab, die sich mit -46.30 bzw. -84.59 ppm (Tab. 5) auf 100 bzw. $96 \%$ derjenigen bei 1 belaufen. Würde die Ladung in der Phenylgruppe von der Wechselwirkung mit der Vinylenbrücke herrühren, dann müßten die Deprotonierungseffekte auf C-2,4 und C-6,7 wegen der verstärkten Homokonjugation größere Absolutwerte als bei 1 aufweisen.

Spektroskopische Daten und Verhalten von 22 widerlegen auch die Behauptung, die besondere Stabilisierung von 1 werde durch den induktiven Effekt der Vinylenbrücke, $d$. $h$. durch zwei elektronenanziehende $\mathrm{sp}^{2}$-C-Atome $\beta$-ständig $\mathrm{zu}$ den Zentren der anionischen Ladung bewirkt ${ }^{13}$. Träfe dies zu, dann müßte 22 relativ zu 1 beachtlich stabilisiert sein, und zwar mindestens um die Hälfte des Betrags, um den $1 \mathrm{im}$ Vergleich zum Anion von 3 energieärmer ist, weil 22 über ein weiteres $\mathrm{sp}^{2}$-C-Atom (das ipso-C der Phenylgruppe) $\beta$-ständig zu den Zentren der negativen Ladung verfügt. Obwohl wir den $\mathrm{p} K_{\mathrm{a}}$-Wert von 16 nicht bestimmt haben, so spricht doch die geringe Haltbarkeit der NMR-Probe von 22 (vgl. Abschnitt B 3) gegen eine im Vergleich zu 2 wesentlich erhöhte Acidität, die derjenigen von 19 und 20 angenähert sein müßte. Natürlich sollte eine solche Stabilisierung eine entsprechende Entschirmung von C-6,7 zur Folge haben, was nicht der Fall ist.

Der besonderen Aufmerksamkeit bedürfen die Deprotonierungseffekte auf C-8. Bei $2 \rightarrow 1$ beträgt dieser Wert -9.07 ppm (Tab. 5). Er ist nicht auf den Ringstrom des homoaromatischen Systems zurückzuführen, denn in der ${ }^{13} \mathrm{C}$-NMR-Spektroskopie sind Anisotropieeffekte von Aromaten relativ zu anderen Beiträgen zur chemischen Verschiebung so gering, $\mathrm{da} B$ sie keine diagnostische Bedeutung haben ${ }^{17 d)}$. Sie fallen in etwa gleich groß aus wie die Einflüsse auf die chemischen Verschiebungen der an die betreffenden C-Atome gebundenen H-Atome. Nun sind aber die Signale von 8- $\mathrm{H}_{2}$ in $1 \mathrm{im}$ Vergleich zu 2 nur um $1.06 \mathrm{bzw} .1 .31 \mathrm{ppm}$ nach höherem Feld verändert.

Was als Ringstromeffekt erwartet werden darf, macht das bishomoaromatische Dianion von 1,6-Dimethylbicyclo[4.4.1]undeca-2,4,7,9-tetraen klar, in dessen

Chem. Ber. 119 (1986) 
Spektren verglichen mit denen des neutralen Tetraens das Signal von $11-\mathrm{H}_{2}$ um $3.3 \mathrm{ppm}$ und das von C-11 um $1.8 \mathrm{ppm}$ nach höherem Feld verschoben sind ${ }^{40)}$.

Tab. 5 legt nahe, daß die Deprotonierungseffekte auf C-8 nichts mit dem homoaromatischen Charakter der Bicyclo[3.2.1]octadienyl-Anionen zu tun haben, denn die Werte variieren nur geringfügig $(-9.07 \mathrm{bis}-6.00 \mathrm{ppm}$ ). Die unter $1 \mathrm{ppm}$ liegenden Werte von 21 mit Bezug auf 19b bleiben außer Betracht, weil sie den $\gamma$-gauche-Effekt der exo-Phenylgruppe auf C-8 in $19 \mathrm{~b}$ enthalten. Mit großer Wahrscheinlichkeit ist die Abschirmung von C-8 eine inhärente Eigenschaft des in der Briefumschlagkonformation fixierten 2-Cyclohexen-1-yl-Anion-Strukturelements. Die elektronische Verwandtschaft zu starren 1,3-Cycloheptadien-Derivaten analoger Konformation liegt auf der Hand, und dort wurden systematische Abschirmungen von C-6 relativ zu hydrierten Vergleichsverbindungen beobachtet, etwa in Bicyclo[4.1.1] octa-2,4-dien-Abkömmlingen ${ }^{41,42 b)}$ und in Octavalen ${ }^{42}$.

Der Eindruck, da $B$ sich die Deprotonierungseffekte auf C-1,5 (Tab. 5) sprunghaft $(+6.09$ bis $-4.92 \mathrm{ppm})$ von Fall zu Fall ändern, trügt, denn die Werte mit negativem Vorzeichen, die ausschließlich bei einer Phenylgruppe in 4-Position des betreffenden Kohlenwasserstoffs gefunden werden, sind der Änderung des $\beta$-Substituenteneffekts der Phenylgruppe auf das benachbarte Brückenkopf-C-Atom bei Abspaltung des Protons von C-4 anzulasten. Als Ursache für die positiven Werte bei $2 \rightarrow 1,4 \rightarrow 5$ und $16 \rightarrow 22$ kann man den elektrischen Feldeffekt ${ }^{17 e)}$ der negativen Ladung der Anionen in Betracht ziehen.

\section{Schlußbetrachtung}

Der bishomoaromatische Charakter des Bicyclo[3.2.1]octadienyl-Anions (1) wird am besten durch Formel 25 repräsentiert, welche die Delokalisierung der negativen Ladung über die Positionen 2, 4, 6 und 7 durch Wechselwirkung zwischen Allyl-Anion-Teil und Vinylenbrücke beschreibt. An C-3 tritt eine negative Partialladung nicht auf (vgl. 22).

Phenylsubstituenten am Allylteil und Benzoanellierung an C-6,7 schwächen aus verschiedenen Gründen die Homokonjugation, heben sie aber selbst beim 2,4Diphenylderivat 7 nicht ganz auf. Im Gegensatz zur Ansicht anderer Autoren ${ }^{9,13)}$ beantworten wir die Frage nach der Homoaromatizität von Bicyclo[3.2.1] octadienyl-Anionen prinzipiell mit ja. Dabei muß aber beachtet werden, $\mathrm{da} B$ die zugehörige Stabilisierungsenergie, die in $1 \mathrm{mit}$ mindestens $12 \mathrm{kcal} / \mathrm{mol}^{11}$ das Maximum erreicht, stark variationsfähig ist, je nachdem inwieweit Substituenten oder Gegenion die negative Ladung mitzutragen vermögen. Formel 25 läßt voraussehen, da $B$ Phenylgruppen an C-6,7 das Ausma $B$ der homokonjugativen Wechselwirkung über jenes in 1 steigern sollten.<smiles>C1C2CC3CC1CC(C2)C3</smiles><smiles>C1=CC2CC1C2</smiles>

Anders als Trimitsis und Zimmerman ${ }^{9 c)}$ erachten wir aufgrund der Ausführungen in Abschnitt $\mathrm{C}$ die ${ }^{13} \mathrm{C}$-chemischen Verschiebungen als sehr leistungsfähiges

Chem. Ber. 119 (1986) 
Kriterium zur Beurteilung von Fernwechselwirkungen zwischen $\pi$-Elektronensystemen in Carbanionen. Alternative Interpretationen der ${ }^{13} \mathrm{C}-\mathrm{NMR}$-Daten, etwa die Deutung der Abschirmung von C-6,7 in 1 als Folge einer Komplexierung des endo-seitig angeordneten Kalium-Ions durch Allyl-Anion- und Vinylenteil ohne deren gegenseitige Wechselwirkung, haben keinerlei Vorbilder und erscheinen unter dem Eindruck der vorgestellten Reihe von 1-Derivaten nicht begründet.

So bleibt noch die Frage zu beantworten, warum im Bicyclo[3.2.1] octadienylRadikal 26 keine Wechselwirkung zwischen den $\pi$-Elektronensystemen besteht, was man aus den kleinen Hyperfeinkopplungskonstanten von 6,7- $\mathrm{H}$ mit dem Einzelelektron wei ${ }^{43}$. Da die Stabilisierung durch Homokonjugation in 1 auf der Mischung des $\varphi_{2}$-Orbitals des Allyl-Anion-Teils mit dem $\pi^{*}$-Orbital der Vinylenbrücke beruht, wird sie mit einer Erhöhung der Spannungsenergie erkauft, weil im Vergleich zur Geometrie ohne Homokonjugation die Bindungsbeziehungen C-2/C-7 und C-4/C-6 naturgemäß zu einer Verkürzung der Abstände zwischen diesen Zentren führen. Verglichen mit 1 fehlt aber im HOMO des Radikals 26 eines der beiden für die nichtklassische Wechselwirkung entscheidenden Elektronen (vgl. Abb. 2). Somit ist die elektronische Stabilisierung auf die Hälfte derjenigen von 1 reduziert und reicht im Gegensatz zu dort nicht mehr aus, um die in Kauf zu nehmende Spannungsenergie-Erhöhung zu kompensieren.

Wir danken der Deutschen Forschungsgemeinschaft und dem Fonds der Chemischen Industrie für die Förderung.

\section{Experimenteller Teil}

Geräte: siehe Lit. $^{42 b)}$.

\section{A. Darstellung der Vorstufen für substitujerte Bicyclo[3.2.1] octadienyl-Anionen}

exo-6-Brom-[2,4-D $D_{2}$ tricyclo[3.2.1.0 $\left.0^{2.7}\right]$ oct-3-en (11 a) und die Monodeuterioderivate 11 b,c: Entsprechend der Vorschrift für die Umsetzung von $2^{22)}$ wurde $\left[2,4,4-D_{3}\right]-2^{63)}$ mit einem Anteil weniger deuterierten Materials mit $N$-Bromsuccinimid zur Reaktion gebracht. Man erhielt mit $28 \%$ Ausb. ein Gemisch aus 11 a, b, c im Verhältnis 8:1:1. - ${ }^{1} \mathrm{H}-\mathrm{NMR}$ : Im Vergleich zu den Angaben in Lit. ${ }^{22}$ ist das Spektrum aufgrund der Deuteriumatome erwartungsgemäß vereinfacht. $-{ }^{13} \mathrm{C}-\mathrm{NMR}$ : Schema 1.

endo-6-Methoxy-[2,4-D $D_{2}$ tricyclo[3.2.1.0 $\left.{ }^{2,7}\right]$ oct-3-en (12a), exo-4-Methoxy-[2.4- $\left.D_{2}\right]$ bicyclo[3.2.1 Jocta-2,6-dien (13a) und die Monodeuterioderivate 12b,c, 13b,c sowie die Diastereomeren von $13 \mathrm{a}$-c: $2.30 \mathrm{~g}$ (13.5 mmol) fein verriebenes $\mathrm{AgNO}_{3}$ (oder $\mathrm{AgBF}_{4}$ ) wurden in $25 \mathrm{ml}$ wasserfreiem Methanol suspendiert und 30 min gerührt, bevor man bei $20^{\circ} \mathrm{C} 2.30 \mathrm{~g}$ (12.3 mmol) 11 zugab. Es schied sich sofort ein gelblich-grüner Niederschlag ab. Man rührte $16 \mathrm{~h}$ unter LichtausschluB, filtrierte $\mathrm{ab}$, wusch sorgfältig mit Methanol nach, fälte restliche $\mathrm{Ag}^{+}$-Ionen im Filtrat mit $20 \mathrm{ml}$ wäBrigem $\mathrm{NaCl}$, filtrierte erneut, extrahierte das Filtrat zweimal mit je $50 \mathrm{ml}$ Ether, wusch die vereinigten organischen Phasen mit wäßriger $\mathrm{NaHCO}_{3}$-Lösung und dann mit Wasser und trocknete über $\mathrm{K}_{2} \mathrm{CO}_{3}$. Die Hauptmenge des Lösungsmittels wurde über eine kurze Vigreuxkolonne bei Normaldruck abdestilliert. Aus dem Rückstand destillierten bei $30^{\circ} \mathrm{C}$ (Bad) $/ 0.1$ Torr $830 \mathrm{mg}(49 \%)$ einer gelblichen Flüssigkeit, die laut 'H-NMR-Spektrum hauptsächlich aus drei Isomeren bestand. Ihre Trennung erfolgte durch präparative Gaschromatographie (6-m-Säule, 20\% DEGS auf Chromosorb

Chem. Ber. 119 (1986) 
W/AW-DMCS, $170^{\circ} \mathrm{C}$ ). Mit Retentionszeiten von 12,14 und 22 min erhielt man 13a, b, c, die zugehörigen endo-Isomeren und 12a, b,c im Verhältnis 1.5:1.0:1.5. Die Charakterisierung erfolgte anhand der ${ }^{1} \mathrm{H}-\mathrm{NMR}$-Spektren durch Vergleich mit den Daten der nichtdeuterierten Verbindungen ${ }^{23)}$ 'und der ${ }^{13} \mathrm{C}-\mathrm{NMR}$-Spektren (Schema 1).

3-Phenylbicyclo[3.2.1 Joct-6-en-3-ol (15): Zu der aus $2.05 \mathrm{~g}$ (84.3 mmol) Magnesiumspänen und $11.0 \mathrm{~g}$ (70.1 mmol) Brombenzol in $8 \mathrm{ml}$ absol. Ether bereiteten Phenylmagnesiumbromid-Lösung tropfte man bei $0^{\circ} \mathrm{C} 2.15 \mathrm{~g}(17.6 \mathrm{mmol})$ Keton $14^{25-27)}$ und setzte das Rühren zunächst $30 \mathrm{~min}$ bei $0^{\circ} \mathrm{C}$ und dann $4 \mathrm{~h}$ bei $20^{\circ} \mathrm{C}$ fort. Nach Hydrolyse mit $5 \mathrm{ml}$ Eiswasser gab man $3 \mathrm{ml} 2 \mathrm{~N} \mathrm{HCl}$ zu, extrahierte zweimal mit Ether, wusch die vereinigten Etherphasen mit 10proz. $\mathrm{NaHCO}_{3}$-Lösung und trocknete über $\mathrm{Na}_{2} \mathrm{SO}_{4}$. Der nach Verdampfen i. Vak. verbliebene gelbliche kristallisierte Rückstand wurde, in Pentan gelöst, an Aluminiumoxid (Akt.-Stufe III, Säule $10 \times 3 \mathrm{~cm}$ ) chromatographiert. Die eingeengte Pentanlōsung schied $2.90 \mathrm{~g} \mathrm{(82 \% )} 15$ als farblose Kristalle mit Schmp. $90-91^{\circ} \mathrm{C}$ ab. Der Einsatz von Phenyllithium anstelle von Phenylmagnesiumbromid, allerdings im Vergleich $\mathrm{zu}$ oben in der 10fachen Menge Ether, erbrachte $15 \mathrm{mit} 47 \%$ Ausbeute. - MS (70 eV): $\mathrm{m} / \mathrm{z}=200(58 \%$, $\mathrm{M}^{+}$), $105(89), 80(100) .-{ }^{1} \mathrm{H}-\mathrm{NMR}\left(\mathrm{CDCl}_{3}\right): \delta=1.74\left(\mathrm{~d}, J_{8,8}=9.9 \mathrm{~Hz} ; 8-\mathrm{H}_{s y n}\right), 1.97(\mathrm{~m}$, enthält $J_{2 e n d o, 8 a n t i}$ endo-2,4-H), $2.19\left(\mathrm{~m} ;\right.$ exo-2,4-H), 2.21 (dtt; $J_{1,8 a n t l}=5.3, J_{2 e n d o, 8 a n t t}=2.7 \mathrm{~Hz}$; 8-H $\left.\mathrm{H}_{\text {anti }}\right), 2.79$ (m; 1,5-H), 3.46 (s; OH), 6.36 (br. s; 6,7-H); $\mathrm{C}_{6} \mathrm{H}_{5:} 7.19$ (p-H), $7.32(m-\mathrm{H}), 7.46$ $(0-\mathrm{H}) .-{ }^{13} \mathrm{C}-\mathrm{NMR}\left(\mathrm{CDCl}_{3}\right): \delta=39.06$ (d; C-1,5), 44.17 (t; C-2,4), 45.09 (t; C-8), 74.64 (s; C-3), 138.37 (d; C-6,7), $\mathrm{C}_{6} \mathrm{H}_{5}: 125.06$ und 127.91 (o- und $m-\mathrm{C}$ ), 126.09 (p-C), 149.80 (ipso-C).

$$
\mathrm{C}_{14} \mathrm{H}_{16} \mathrm{O}(200.3) \text { Ber. C } 83.96 \text { H } 8.05 \text { Gef. C } 84.00 \text { H } 8.28
$$

3-Phenylbicyclo/3.2.1 ]octa-2,6-dien (16): $4.00 \mathrm{~g}$ (20.0 mmol) 15 und $9.56 \mathrm{~g}$ (40.1 mmol) $\left(\mathrm{C}_{2} \mathrm{H}_{5}\right)_{3} \mathrm{~N}^{\oplus} \mathrm{SO}_{2} \mathrm{~N}^{\ominus} \mathrm{CO}_{2} \mathrm{CH}_{3}{ }^{28)}$ wurden in $50 \mathrm{ml}$ Benzol $30 \mathrm{~min}$ bei $50^{\circ} \mathrm{C}$ gerührt. Man gab dann $50 \mathrm{ml}$ Wasser zu, extrahierte zweimal mit je $20 \mathrm{ml}$ Ether, wusch die vereinigten organischen Phasen zweimal mit je $50 \mathrm{ml}$ Wasser und trocknete über $\mathrm{Na}_{2} \mathrm{SO}_{4}$. Nach $\mathrm{Ab}$ dampfen der Lösungsmittel i. Vak. destillierten bei $80-100^{\circ} \mathrm{C}(\mathrm{Bad}) / 0.1$ Torr $1.50 \mathrm{~g}(42 \%)$ 16 als farblose Flüssigkeit aus dem Rückstand. - MS (70 eV): $m / z=182\left(69 \%, M^{+}\right), 104$ (100). - ${ }^{1} \mathrm{H}$ - und ${ }^{13} \mathrm{C}-\mathrm{NMR}$ : Tab. 1, 2.

$$
\mathrm{C}_{14} \mathrm{H}_{14} \text { (182.3) Ber. C } 92.26 \text { H } 7.74 \text { Gef. C } 92.32 \text { H } 7.62
$$

endo-6-[(Z)-2-Phenylvinyl]bicyclo[3.1.0] hex-2-en (18b), endo- (19a) und exo-4-Phenylbicyclo[3.2.1 jocta-2,6-dien (19b): Zur Lösung von $7.00 \mathrm{~g}$ (304 mmol) Natrium in $600 \mathrm{ml}$ absol. Ethanol fügte man bei $0^{\circ} \mathrm{C}$ unter Rühren portionsweise $78.0 \mathrm{~g}(201 \mathrm{mmol})$ Benzyltriphenylphosphoniumchlorid. Nach vollendeter Zugabe rührte man noch $30 \mathrm{~min}$ bei $0^{\circ} \mathrm{C}$, tropfte dann bei $0^{\circ} \mathrm{C} 20.0 \mathrm{~g}(185 \mathrm{mmol})$ des Aldehyds $17^{44)}$ in $80 \mathrm{ml}$ Ethanol ein und rührte $2 \mathrm{~h}$ bei $20^{\circ} \mathrm{C}$. Danach erfolgten Hydrolyse, dreimalige Extraktion mit je $100 \mathrm{ml}$ Ether und Trocknen der vereinigten organischen Phasen über $\mathrm{Na}_{2} \mathrm{SO}_{4}$. Man verdampfte die Lösungsmittel i. Vak. und erhielt aus dem Rückstand durch Destillation bei $80^{\circ} \mathrm{C}(\mathrm{Bad}) / 0.1$ Torr $9.36 \mathrm{~g}(28 \%)$ einer farblosen Flüssigkeit, deren NMR-Spektren das Vorliegen von 19a und 18b im Verhältnis $2: 3$ anzeigten. Erhitzen dieses Gemisches in Substanz auf $150^{\circ} \mathrm{C}$ erbrachte nach $5 \mathrm{~h}$ ein 2:3-Gemisch aus 19a und 19b, das wieder bei 0.1 Torr destilliert wurde. Durch fraktionierte Destillation des $18 \mathrm{~b} / 19 \mathrm{a}-$ Gemisches isolierte man eine geringe Menge fast reines 18b. $-{ }^{1} \mathrm{H}-\mathrm{NMR}\left(\mathrm{CDCl}_{3}\right): 18 \mathrm{~b}: \delta=1.97\left(\approx \mathrm{q}, J_{1,5}=6.0, J_{5,6}=8.0 \mathrm{~Hz} ; 5-\mathrm{H}\right), 2.01\left(\mathrm{q}, J_{1,6}=\right.$ $\left.J_{6,1^{\prime}}=8.1 \mathrm{~Hz} ; 6-\mathrm{H}\right), 2.22\left(\mathrm{~d}, J_{4,4}=18.5 \mathrm{~Hz} ; 4-\mathrm{H}_{\text {endo }}\right), 2.29(\mathrm{~m} ; 1-\mathrm{H}), 2.57$ (ddt, $J_{\text {4exo }, 5}=7.0$, $\left.J_{2 \text { eexo }}=J_{3, \text { Aexo }}=2.0 \mathrm{~Hz} ; 4-\mathrm{H}_{\text {exo }}\right), 5.64(\mathrm{dm} ; 3-\mathrm{H}), 5.69\left(\mathrm{dq}, J_{2,3}=5.6 ; J_{1,2}=J_{2 \text {,endo }}=\right.$ $2.0 \mathrm{~Hz} ; 2-\mathrm{H}), \mathrm{CH}=\mathrm{CHC}_{6} \mathrm{H}_{5}: 5.36\left(\mathrm{dd}, J_{1 \cdot 2^{\prime}}=11.8 \mathrm{~Hz} ; 1^{\prime}-\mathrm{H}\right), 6.49\left(\mathrm{~d} ; 2^{\prime}-\mathrm{H}\right), 7.19(p-\mathrm{H}), 7.31$ $(m-H), 7.45(o-H) .19 a$, b: Tab. 1. $-{ }^{13} \mathrm{C}-\mathrm{NMR}\left(\mathrm{CDCl}_{3}\right):$ 18b: $\delta=21.17,23.98,32.23,32.59$,

Chem. Ber. 119 (1986) 
126.37, 126.85, 128.04 und $128.67(o, m-C), 129.10,131.04,131.26,138.16$ (ipso-C). 19a, b: Tab. 2

$$
\begin{array}{llll}
\mathrm{C}_{14} \mathrm{H}_{14} \text { (182.3) } & \text { Ber. C } 92.26 \text { H } 7.74 \begin{array}{ll}
\text { 19a } \\
\text { 20: }
\end{array} & \text { 19b: } & \text { Gef. C } 92.34 \text { H } 7.59 \\
\text { Gef. C } 92.57 \text { H } 7.45
\end{array}
$$

2-Phenylbicyclo[3.2.1]octa-2,6-dien (20): Man rührte $500 \mathrm{mg}(2.74 \mathrm{mmol}) 19 \mathrm{a}, \mathrm{b}$ und $200 \mathrm{mg}$ (1.78 mmol) Kalium-tert-butoxid in $3 \mathrm{ml}$ absol. Dimethylsulfoxid bei $20^{\circ} \mathrm{C}$. Nach 30 min setzte man je $5 \mathrm{ml}$ Wasser und Pentan zu, extrahierte die wäßrige Phase zweimal mit je $5 \mathrm{ml}$ Pentan und dann die vereinigten Pentanphasen zweimal mit je $10 \mathrm{ml}$ gesättigter $\mathrm{NaCl}$-Lösung, trocknete über $\mathrm{Na}_{2} \mathrm{SO}_{4}$ und engte i. Vak. ein. Aus dem Rückstand destillierten bei $80-100^{\circ} \mathrm{C}(\mathrm{Bad}) / 0.1$ Torr $320 \mathrm{mg}(64 \%) 20$ als farblose Flüssigkeit. - 'H-NMR: Tab. 1.

2-Phenyl-[4,4- $D_{2}$ ]bicyclo[3.2.1]octa-2,6-dien ( $\left.\left[\mathrm{D}_{2}\right]-20\right)$ ): Als man im Vergleich zum vorangehenden Versuch $\left[D_{6}\right]$ Dimethylsulfoxid als Lösungsmittel verwendete, fehiten im ' $\mathbf{H}$ NMR-Spektrum des Produkts die Signale bei $\delta=1.95$ und 2.45 , und die Signale bei $\delta=$ $5.60(3-\mathrm{H})$ und $2.73(5-\mathrm{H})$ waren entsprechend vereinfacht.

\section{B. Bereitung der substituierten Bicyclo[3.2.1]octadienyl-Anionen}

Alle Operationen wurden unter strengem AusschluB von Feuchtigkeit und Sauerstoff durchgeführt. Als Schutzgas diente entsprechend präpariertes Argon.

2,4-Diphenylbicyclo[3.2.1 Jocta-3,6-dien-2-yllithium (7): In einem 5-ml-Kölbchen mit seitlich angeschmolzenem NMR-Röhrchen engte man i. Vak., zuletzt $0.001 \mathrm{Torr}, 0.55 \mathrm{ml} 1.5 \mathrm{~N}$ Methyllithium $(0.83 \mathrm{mmol})$ in Ether unter Rühren zur Trockne ein. Der Rückstand wurde in $0.5 \mathrm{ml}\left[\mathrm{D}_{8}\right]$ Tetrahydrofuran aufgenommen und das Gemisch im Eisbad gekühlt. Dann tropfte man $200 \mathrm{mg}(0.77 \mathrm{mmol}) 6$, gelöst in $0.5 \mathrm{ml}\left[\mathrm{D}_{8}\right]$ Tetrahydrofuran, aus einer Spritze zu, wobei sehr rasch die rote Farbe von 7 auftrat. Man rührte die tiefrote Lösung $2 \mathrm{~h}$ bei $20^{\circ} \mathrm{C}$, goß sie in das NMR-Röhrchen und schmolz dieses i. Vak. ab. Neben 7 als weit überwiegender Spezies zeigten die NMR-Spektren (Tab. 3 und 4) einige Verunreinigungen an, darunter ca. $15 \%$ Ether, bezogen auf 7.

2-Phenylbicyclo[3.2.1 ]octa-3,6-dien-2-yllithium (21 Li): Unter Verwendung von $0.56 \mathrm{mmol}$ $n$-Butyllithium in Ether anstelle von Methyllithium erhielt man aus $100 \mathrm{mg}(0.55 \mathrm{mmol})$ $19 \mathrm{a}$, b wie bei 7 beschrieben eine rotbraune Lösung von $21 \mathrm{Li}$ in $\left[\mathrm{D}_{8}\right]$ Tetrahydrofuran. Das ${ }^{1}$ H-NMR-Spektrum (Tab. 3) zeigte neben starken Ether-Banden zwei intensive Multipletts $\delta=0.82$ und 1.22. Diese Signale überlagerten die 8- $\mathrm{H}_{2}-$ Banden von $21 \mathrm{Li}$, während dessen weitere Absorptionen völlig frei lagen. Aus dem ${ }^{13} \mathrm{C}$-NMR-Spektrum folgt, daB der Etheranteil, bezogen auf $21 \mathrm{Li}$, ca. $50 \%$ betrug, und die andere Verunreinigung mengenmäßig weit weniger bedeutend war. Die ungewöhnlich große Linienbreite einiger Signale im Routine${ }^{13} \mathrm{C}$-NMR-Spektrum zeigte einen langsamen dynamischen Vorgang an, der durch Tieftemperaturmessungen genauer untersucht wurde (siehe Tab. 4).

2-Phenylbicyclo[3.2.1 Jocta-3,6-dien-2-ylkalium (21 K): Als Reaktionsgefäß diente ein 50ml-Rundkolben mit verlängertem Hals und den drei folgenden Ansätzen: 1) am Kolben schräg nach oben ein NMR-Röhrchen; 2) diesem gegenüber wieder schräg nach oben eine Fritte, daran ein Hahn und dann ein Schlifkern zum Aufsetzen eines Kölbchens; 3) am verlängerten Hals (zwischen Kolben und Schliff) horizontal und in einer Ebene senkrecht zur Ebene der beiden anderen Ansätze wieder ein Hahn und daran ein Schliff zum Anschluß an eine Vakuumlinie.

Man legte in diesem Kolben $170 \mathrm{mg}$ (1.52 mmol) Kalium-tert-butoxid in ca. $5 \mathrm{ml}$ Pentan vor, kühlte das Gemisch auf $-78^{\circ} \mathrm{C}$ und tropfte mit einer Spritze durch eine Serumkappe unter Rühren zuerst $1.50 \mathrm{mmol} n$-Butyllithium (1.65 ml, $0.9 \mathrm{~N}$ in Ether) und dann $90 \mathrm{mg}$

Chem. Ber. 119 (1986) 
(0.49 mmol) 19a, b in $1 \mathrm{ml}$ Pentan zu. Man entfernte das Kühlbad und ließ die Suspension auf $20^{\circ} \mathrm{C}$ kommen, wobei sie rasch eine orangerote Farbe annahm. Nach 24 h Rühren bei $20^{\circ} \mathrm{C}$ befand sich ein rotbrauner Niederschlag in einer tiefroten Lösung. Durch vorsichtiges Kippen der Apparatur wurde die Lösung durch die Fritte entfernt. Man gab dann $10 \mathrm{ml}$ Pentan zum Rückstand, rührte 30 min, dekantierte nach Absitzen des Niederschlags erneut und wiederholte diese Arbeitsgänge noch zweimal. Im $10^{-3}$-Torr-Vakuum wurde dann das restliche Lösungsmittel entfernt. Die metallorganische Verbindung $21 \mathrm{~K}$ verblieb dabei als dunkel rotbrauner Belag an der Kolbenwand. Man kühlte den Kolben nun mit flüssigem Stickstoff und kondensierte $0.5 \mathrm{ml}\left[\mathrm{D}_{8}\right]$ Tetrahydrofuran von Lithiumaluminiumhydrid ab durch die Vakuumlinie auf $21 \mathrm{~K}$. Nach Erwärmen auf $20^{\circ} \mathrm{C}$ löste man den Niederschlag durch Schwenken des Kolbens auf, füllte die fast schwarze Lösung in das NMR-Röhrchen und schmolz nach nochmaligem Entgasen i. Vak. ab. Im ${ }^{1} \mathrm{H}-\mathrm{NMR}$-Spektrum (Tab. 3) zeigen intensive Banden bei $\delta=0.8-1.6$ Verunreinigungen an. Diese Banden überlagern die 8- $\mathrm{H}_{2}$-Signale von $21 \mathrm{~K}$. Im ${ }^{13} \mathrm{C}$-NMR-Spektrum (Tab. 4) stören die Verunreinigungen nicht, denn von ihnen stammen nur drei Linien bei $\delta=14.92,20.50$ und 36.91, deren Intensitāten denen der $21 \mathrm{~K}$-Linien vergleichbar sind und die sich auch in den Spektren von 5 und 22 finden. Die Routine ${ }^{13} \mathrm{C}$-NMR-Messungen, bei denen unter Breitbandentkopplung und ohne Entkopplung verschiedene Temperaturen herrschten, gaben im Gegensatz zur Messung von $21 \mathrm{Li}$ keinen Hinweis auf eine Temperaturabhängigkeit der Spektren.

Tricyclo[6.3.1.0 2.7] dodeca-2,4,6,10-tetraen-9-ylkalium (5): Wie bei $21 \mathrm{~K}$ beschrieben, brachte man $337 \mathrm{mg}$ (3.00 mmol) Kalium-tert-butoxid und $300 \mathrm{mmol} n$-Butyllithium mit $156 \mathrm{mg}(1.00 \mathrm{mmol}) 4^{15)}$ zur Reaktion. Nachdem die rotbraune $\left[\mathrm{D}_{8}\right.$ ] Tetrahydrofuran-Lösung von $524 \mathrm{~h}$ bei $-20^{\circ} \mathrm{C}$ aufbewahrt worden war, zeigte ihr ${ }^{1} \mathrm{H}-\mathrm{NMR}$-Spektrum schon deutliche Signale von [4-D]-4. Tab. 3 und 4 enthalten die Daten der bei $-50^{\circ} \mathrm{C}$ gemessenen Spektren, die auf die gleichen Verunreinigungen hinweisen wie bei $21 \mathrm{~K}$.

3-Phenylbicyclo[3.2.1]octa-3,6-dien-2-ylkalium (22): Wie bei $21 \mathrm{~K}$ beschrieben, brachte man $170 \mathrm{mg}(1.52 \mathrm{mmol})$ Kalium-tert-butoxid und $1.53 \mathrm{mmol} n$-Butyllithium mit $90 \mathrm{mg}$ (0.49 mmol) 16 zur Reaktion. Die NMR-Spektren der dunkelbraunen $\left[D_{8}\right]$ Tetrahydrofuranlösung von 22 wurden bei $-50^{\circ} \mathrm{C}$ gemessen (Tab. 3 und 4). Es fanden sich die gleichen Verunreinigungen wie bei $21 \mathrm{~K}$. Aufbewahren bei $-78^{\circ} \mathrm{C}$ konnte die Zersetzung der Probe, die sich in einer Viskositätszunahme und im Auftauchen breiter ${ }^{1} \mathrm{H}-\mathrm{NMR}$-Signale bei $\delta$ 프 4.5-6.3 äußerte, nicht verhindern.

\section{CAS-Registry-Nummern}

1: $101165-91-7$ / 2: 4096-95-1 / 2-(2,4,4-D $): 101166-04-5$ / 4: 24309-43-1 / 5: 101165-92-8 / 6: 57542-07-1 /7: 101165-93-9 / 11a: 101165-95-1 / 11 b: 101165-96-2 / 11 c: 101165-97-3 / 12a: 101166-01-2 / 12b: 101166-02-3 / 12c: 101166-03-4 / 13a: 101165-98-4 / 13b: $101165-$ 99-5 / 13c: 101166-00-1 / 14: 3721-60-6 / 15: 101166-05-6 / 16: 101166-06-7 / 17: 472905-9 / 18b: 101166-07-8 / 19 a: 101165-89-3 / 19b: 101165-90-6 / 20: 101166-08-9 / 20-4,4$\left.\mathrm{D}_{2}\right)$ : 101166-09-0 / 21 Li: 101166-10-3 / 21 K: 101166-11-4 / 22: 101165-94-0/ $\mathrm{Et}_{3} \mathrm{~N}^{\oplus}$ $\mathrm{SO}_{2} \mathrm{~N}^{\Theta} \mathrm{CO}_{2} \mathrm{Me}: 29684-56-8 / \mathrm{D}_{2}: 7782-39-0$

\footnotetext{
${ }^{1)}$ Aus der Dissertation D. Brückner, Univ. Würzburg 1985.

2) 2a) S. Winstein, Spec. Publ. Chem. Soc. No. 21, $5(1967)$. - 2b) P. M. Warner, Top. Nonbenzenoid Aromatic Chem. 1976, 2. - ${ }^{20}$ L. A. Paquette, Angew. Chem. 90, 114 (1978); Angew. Chem., Int. Ed. Engl. 17, 106 (1978). - ${ }^{20}$ R. F. Childs, Acc. Chem. Res. 17, 347 (1984).

3) 3a) R. C. Haddon und K. Raghavachari, J. Am. Chem. Soc. 105, 118 (1983). - ${ }^{36)}$ D. Cremer,

E. Kraka, T. S. Slee, R. F. W. Bader, C. D. H. Lau, T. T. Nguyen-Dang und P. J.
}

Chem. Ber. 119 (1986) 
MacDougall, J. Am. Chem. Soc. 105, 5069 (1983). - ${ }^{3 c)}$ K. N. Houk, R. W. Gandour, R. W. Strozier, N. G. Rondan und L. A. Paquette, J. Am. Chem. Soc. 101, 6797 (1979).

4) H. Quast und J. Christ, Angew. Chem. 96, 606 (1984); Angew. Chem. Int. Ed. Engl. 23, 631 (1984); H. Quast, E. Geißler, A. Mayer, L. M. Jackman und K. L. Colson, Tetrahedron 42, 1805 (1986).

5) R. Hoffmann und W.-D. Stohrer, J. Am. Chem. Soc. 93, 6941 (1971); M. J. S. Dewar, Z. Náhlovská und B. D. Náhlovský, J. Chem. Soc. D (Chem. Commun.) 1971, 1377; M. J. S. Dewar und D. H. Lo, J. Am. Chem. Soc. 93, 7201 (1971).

๑) 63) J. M. Brown und J. L. Occolowitz, Chem. Commun. 1965, 376; J. Chem. Soc. B 1968, 411. - ${ }^{6 b)}$ J. M. Brown, Chem. Commun. 1967, 638

${ }^{7}$ S. Winstein, M. Ogliaruso, M. Sakai und J. M. Nicholson, J. Am. Chem. Soc. 89, 3656 (1967).

8) J.W. Rosenthal und S. Winstein. Tetrahedron Lett. 1970, 2683; J. M. Brown, E. N. Cain und M. McIvor, J. Chem. Soc. B 1971, 730.

9) 9a) G. B. Trimitsis und A. Tuncay, J. Am. Chem. Soc. 97,7193 (1975). - 96) G. B. Trimitsis und A. Tuncay, J. Am. Chem. Soc. 98, $1997(1976)$. - ${ }^{9 c)}$ G. B. Trimitsis und P. Zimmerman, J. Chem. Soc., Chem. Commun. 1984, 1506.

10\} 10a) M. V. Moncur und J. B. Grutzner, J. Am. Chem. Soc. 95,6449 (1973). - ${ }^{106)}$ M. J. Goldstein und S. Natowsky. J. Am. Chem. Soc. 95, 6451 (1973). - ${ }^{100)} S$. W. Staley und D. W. Reichard, J. Am. Chem. Soc. 91, 3998 (1969).

11) W. N. Washburn, J. Org. Chem. 48, 4287 (1983).

12) A. Streitwieser jr. und $D$. W. Boerth, J. Am. Chem. Soc. 100, 755 (1978)

13) J. B. Grutzner und W. L. Jorgensen, J. Am. Chem. Soc. 103, 1372 (1981); E. Kaufmann, H. Mayr, J. Chandrasekhar und P. v. R. Schleyer, ebenda 103, 1375 (1981).

14) J. M. Brown, R. J. Elliot und W. G. Richards, J. Chem. Soc., Perkin Trans. 2 1982, 485.

i5) P. v. R. Schleyer, T. Clark, A. J. Kos, G. W. Spitznagel, C. Rohde, D. Arad, K. N. Houk und N. G. Rondan, J. Am. Chem. Soc. 106, 6467 (1984), und dort zitierte frühere Arbeiten.

16) R. Eidenschink und T. Kauffmann, Angew. Chem. 84, 292 (1972); Angew. Chem., Int. Ed. Engl. 11, 292 (1972); G. Boche und D. Martens, Angew. Chem. 84, 768 (1972); Angew. Chem., Int. Ed. Engl. 11, 724 (1972); T. Kauffmann, Angew. Chem. 86, 715 (1974); Angew. Chem., Int. Ed. Engl. 13, 627 (1974); W. T. Ford und G. F. Luteri, J. Am. Chem. Soc. 99, 5330 (1977).

17) H.O. Kalinowski, S. Berger und S. Braun, ${ }^{13} \mathrm{C}-\mathrm{NMR}$-Spektroskopie, Thieme, Stuttgart 1984. - ${ }^{17 a)}$ S. $88 \mathrm{ff}, 177,244,370$ ff. - ${ }^{17 b)}$ Kapitel 4. - 17c) S. 74. - 17d)' S. $146 .-$ i7e) S. 205.

18) M. J. Goldstein, S. Tomoda und G. Whittaker, J. Am. Chem. Soc. 96, 3676 (1974).

19) ${ }^{199)} D$. H. O'Brien, A. J. Hart und C. R. Russell, J. Am. Chem. Soc. 97,4410 (1975). 196) D. H. O'Brien, C. R. Russell und A. J. Hart, Tetrahedron Lett. 1976, 37.

${ }^{20)} F$. H. Köhler und N. Hertkorn, Chem. Ber. 116, 3274 (1983).

21) $M$. Christl, H. Leininger und D. Brückner, J. Am. Chem. Soc. 105, 4843 (1983).

22) J. Japenga, G. W. Klumpp und J. Stapersma, Tetrahedron 33, 2847 (1977).

23) $W$. Kirmse und $T$. Olbricht, Chem. Ber. 108, 2616 (1975).

${ }^{24)}$ Vgl. Zitate ${ }^{16-18)}$ von Lit. ${ }^{21)}$ sowie J. R. Wesener, D. Moskau und H. Günther, J. Am. Chem. Soc. 107, 7307 (1985)

25) N. A. LeBel und R. N. Liesemer, J. Am. Chem. Soc. 87, 4301 (1965).

${ }^{26)} H$. Bos und G. $W$. Klumpp. Tetrahedron Lett. 1978, 1863.

27) G. W. Klumpp, H. Bos, M. Schakel, R. F. Schmitz und J. J. Vrielink, Tetrahedron Lett. $1975,3429$.

28) E. M. Burgess, H. R. Penton ir. und E. A. Taylor, J. Org. Chem. 38, 26 (1973).

29) C. Cupas, W. E. Watts und P. v. R. Schleyer Tetrahedron Lett. 1964, 2503

30) $W$. Adam, $O$. De Lucchi und $D$. Scheutzow, J. Org. Chem. 46, 4130 (1981).

31) G. W. Klumpp, J.W. F. K. Barnick, A. H. Veefkind und F. Bickelhaupt, Rec. Trav. Chim. Pays-Bas 88, 766 (1969).

${ }^{32)}$ M. P. Schneider und A. Rau, J. Am. Chem. Soc. 101, 4426 (1979).

33) Zur Natur dieser Base siehe L. Lochmann, J. Pospisil und D. Lim. Tetrahedron Lett. 1966, 257; M. Schlosser, J. Organomet. Chem. 8, 9 (1967), M. Schlosser und J. Hartmann, Angew. Chem. 85, 544 (1973); Angew. Chem., Int. Ed. Engl. 12, 508 (1973); M. Schlosser und S. Strunk, Tetrahedron Lett. 25, 741 (1984).

34) F. H. Köhler und N. Hertkorn, Z. Naturforsch. Teil B 38, 407 (1983).

${ }^{35)} E$. Heilbronner und $H$. Bock, Das HMO-Modell und seine Anwendung. Tabellen berechneter und experimenteller Größen, Verlag Chemie, Weinheim 1970.

Chem. Ber. 119 (1986) 
36) J. P. C. M. van Dongen, H. W. D. van Dijkman und M. J. A. de Bie, Rec. Trav. Chim. Pays-Bas 93, 29 (1974).

${ }^{37}$ R. J. Bushby und G. J. Ferber, J. Chem. Soc., Perkin Trans. 2 1976, 1688.

38) $M$. Saunders, L. Telkowski und M. R. Kates, J. Am. Chem. Soc. 99, 8070 (1977).

39) Zur induktiven Stabilisierung von negativen Ladungen durch Phenylgruppen vgl. $S$. $E$. Browne, S. E. Asher, E. H. Cornwall, J. K. Frisoli, L. J. Harris, E. A. Salot, E. A. Sauter, M. A. Trecoske und P. S. Veale jr., J. Am. Chem. Soc. 106, 1432 (1984).

40) W. Huber, K. Müllen, R. Busch, W. Grimme und J. Heinze, Angew. Chem. 94, 294 (1982); Angew. Chem., Int. Ed. Engl. 21, 301 (1982)

41) $W$. Sander und R. Gleiter, Chem. Ber. 118, 2548 (1985)

42) 42a) M. Christl und $R$. Lang. J. Am. Chem. Soc. 104, 4494 (1982). - ${ }^{42 b)}$ M. Christl, $R$. Lang und C. Herzog. Tetrahedron 42, 1585 (1986).

${ }^{43)} R$. Sustmann und $R$. W. Gellert, Chem. Ber. 111, 42 (1978)

4) J. Meinwald, S. S. Labana und M. S. Chadha, J. Am. Chem. Soc. 85, 582 (1963); J. Meinwald S S Labana, L L Labana und G. H: Wahl ir. Tetrahedron Lett. 1965, 1789

45) R. P. Johnson, A. Exarchou, C. W. Jefford und R. C. Hahn, J. Org. Chem. 42, 3758 (1977). 